Algebraic 83 Geometric $\mathcal{T}$ opology

Volume 5 (2005) 135-182

Published: 12 March 2005

ATG

\title{
Algebraic models of Poincaré embeddings
}

\author{
Pascal Lambrechts \\ Don Stanley
}

\begin{abstract}
Let $f: P \hookrightarrow W$ be an embedding of a compact polyhedron in a closed oriented manifold $W$, let $T$ be a regular neighborhood of $P$ in $W$ and let $C:=\overline{W \backslash T}$ be its complement. Then $W$ is the homotopy push-out of a diagram $C \leftarrow \partial T \rightarrow P$. This homotopy push-out square is an example of what is called a Poincaré embedding.

We study how to construct algebraic models, in particular in the sense of Sullivan, of that homotopy push-out from a model of the map $f$. When the codimension is high enough this allows us to completely determine the rational homotopy type of the complement $C \simeq W \backslash f(P)$. Moreover we construct examples to show that our restriction on the codimension is sharp.

Without restriction on the codimension we also give differentiable modules models of Poincaré embeddings and we deduce a refinement of the classical Lefschetz duality theorem, giving information on the algebra structure of the cohomology of the complement.
\end{abstract}

AMS Classification 55P62; 55M05, 57Q35

Keywords Poincaré embeddings, Lefschetz duality, Sullivan models

\section{Introduction}

Let us recall the notion of a Poincaré embedding:

Definition 1.1 (Levitt [23], and [15, Section 5] for a modern exposition) Let $W$ be a Poincaré duality space of dimension $n$ and let $P$ be a finite CWcomplex of dimension $m$. A Poincaré embedding of $P$ in $W$ (of dimension $n$ and codimension $n-m$ ) is a commutative diagram of topological spaces

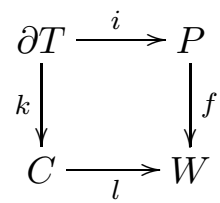


such that (1.1) is a homotopy push-out, $(P, \partial T)$ and $(C, \partial T)$ are Poincaré duality pair 1 in dimension $n$, and the map $i$ is $(n-m-1)$-connected.

The motivating example of a Poincaré embedding arises when $W$ is a closed orientable PL-manifold of dimension $n$ and $f: P \hookrightarrow W$ is a piecewise linear embedding of a compact polyhedron $P$ in $W$. Alternatively we can also take $f$ to be a smooth embedding between smooth compact manifolds. Then $f(P)$ admits a regular neighborhood, that is a codimension 0 compact submanifold $T \subset W$ that deformation retracts to $P$ (see [26, page 33].) Let $C:=\overline{W \backslash T}$ be the closure of the complement of $T$ in $W$. Then $C$ and $T$ are both compact manifolds of dimension $n$ with a common boundary $\partial T=\partial C$ and $W=T \cup_{\partial T}$ $C$. The composition of the inclusion $\partial T \hookrightarrow T$ with the retraction $T \stackrel{\simeq}{\rightarrow} P$ gives a map $i: \partial T \rightarrow P$ and we obtain the pushout (1.1). If the polyhedron $P$ is of dimension $m$, then a general position argument implies that the map $i$ is $(n-m-1)$-connected. Of course $C$ has the homotopy type of the complement $W \backslash f(P)$.

Thus morally a Poincaré embedding is the homotopy generalization of a PL embedding. Notice that, in Definition 1.1, $\partial T$ is just a topological space and not necessarily a genuine boundary of a manifold $T$, and $W$ does not need to be a manifold. Notice also that by a Poincaré embedding we mean all of the diagram (1.1) and not only the map $f$. When such a diagram exists we say that the map $f: P \rightarrow W$ Poincaré embeds. The space $C$ in the push-out diagram is called the complement of $P$.

A natural question is whether the homotopy class of a map $f$ that Poincaré embeds determines the square (1.1) up to homotopy equivalence and in particular the homotopy type of the complement $C$. The answer to this question is negative in general as it can be seen with $W=S^{3}$ and $P=S^{1}$. Indeed all PL-embeddings $f$ : $S^{1} \hookrightarrow S^{3}$ are nullhomotopic but the homotopy type of the complement $C \simeq S^{3} \backslash f\left(S^{1}\right)$ can vary considerably (see for example [24, Corollary 11.3] or [9.) This is possible since in general the homotopy class $[f]$ of $f$ does not determine its isotopy class. On the other hand in the case of a PL-embedding when the codimension is high enough, namely when $n \geq 2 m+3$, then a general position argument implies that $[f]$ determines the isotopy class of $f$. Therefore under this high codimension hypothesis the homotopy class of a PL-embedding $f$ does determine the homotopy type of the square (1.1). Similarly under a slightly more restrictive condition on the codimension, there exists a unique Poincaré embedding (1.1) associated to a given homotopy class

\footnotetext{
${ }^{1}$ By abuse of terminology, by the pair $(P, \partial T)$ we actually mean the pair $\left(P^{\prime}, \partial T\right)$ where $P^{\prime}$ is the mapping cylinder of $i$, and similarly for the pair $(C, \partial T)$
} 
$[f]$. See Theorem 1.3 below for a precise and more general statement for PLembeddings as well as a discussion on the corresponding result for Poincaré embeddings.

The aim of this paper is to study an algebraic translation of the above question: can we build algebraic models, such as Sullivan models which encode rational homotopy type, of the square (1.1) from an algebraic model of the map $f$ ? In order to be more precise, we first review Sullivan's theory for modeling rational homotopy types by algebraic models. By a $C D G A, A$, we mean a non-negatively graded algebra over the field $\mathbb{Q}$ of rational numbers that is commutative in the graded sense and endowed with a degree +1 derivation $d: A \rightarrow A$ such that $d^{2}=0$. Sullivan has defined in 27] a contravariant functor from topological spaces to CDGA,

$$
A_{P L}: \text { Top } \rightarrow \text { CDGA, }
$$

mimicking the de Rham complex of differential forms on a smooth manifold. By a $C D G A$ model of a space $X$, we mean a CDGA, $A$, linked to $A_{P L}(X)$ by a chain of CDGA morphisms inducing isomorphisms in cohomology,

$$
A \stackrel{\simeq}{\longleftarrow} A_{1} \simeq \cdots \simeq A_{n} \stackrel{\simeq}{\longrightarrow} A_{P L}(X) .
$$

The fundamental result of Sullivan's theory is that if $X$ is a simply-connected space with rational homology of finite type, then any CDGA model of $X$ determines its rational homotopy type. There is a similar result for maps and more generally for finite diagrams. See [8] for a complete exposition of that theory.

Our first result is the construction, under the high codimension hypothesis $\operatorname{dim}(W) \geq 2 \operatorname{dim}(P)+3$, of an explicit CDGA model of the Poincaré embedding (1.1) out of a CDGA-model of $f$. To explain this result, we need some notation which will be made more precise in Section 2 . We denote by $\# V:=\operatorname{hom}(V, \mathbf{k})$ the dual of a k-vector space $V$ and by $s^{p} X$ the $p$-th suspension of a graded object $X$, i.e. $\left(s^{p} X\right)^{k}=X^{p+k}$. The mapping cone of a cochain map $f: M \rightarrow N$ is written $N \oplus_{f} s M$. When $N$ is a CDGA and $M$ is an $N$-DGmodule this mapping cone can be endowed with the multiplication $(n, s m) \cdot\left(n^{\prime}, s m^{\prime}\right)=(n$. $\left.n^{\prime}, s\left(n \cdot m^{\prime} \pm n^{\prime} \cdot m\right)\right)$. The differential of the mapping cone does not always satisfy the Leibnitz rule for this multiplication, but it does under certain conditions on the dimensions and then the induced structure is called the semi-trivial CDGA-structure on the mapping cone (Definition 4.4).

Our goal is to build a CDGA model of the homotopy push-out (1.1), and in particular of the complement $C$, out of a CDGA model $\phi: R \rightarrow Q$ of $f^{*}: A_{P L}(W) \rightarrow A_{P L}(P)$. Motivated by Lefschetz duality a first guess for a 
model of $A_{P L}(C)$ is the mapping cone

$$
R \oplus_{\psi} s s^{-n} \# Q
$$

where

$$
\psi: s^{-n} \# Q \rightarrow R
$$

is an $R$-DGmodule map such that $H^{n}(\psi)$ is an isomorphism. Unfortunately this naive guess has two flaws:

(A) such a map $\psi$ does not necessarily exist, and

(B) the multiplication on $R \oplus_{\psi} s s^{-n} \# Q$ does not necessarily define a CDGA structure because of the possible failure of the Leibnitz rule.

Problem (A) can be addressed by replacing $s^{-n} \# Q$ by a suitable weakly equivalent DG-module $D$, for example a cofibrant one, for which there exists a map $\psi: D \rightarrow R$ inducing an isomorphism in cohomology in degree $n$. Such a map is called a top-degree map 2 in Definition [5.1. Problem (B) can be solved by restricting the range of degrees of the graded objects $R, Q$, and $D$. This is where the high codimension hypothesis is needed.

We can now state our first result:

Theorem 1.2 Consider a Poincaré embedding (1.1) with $P$ and $W$ connected. If $n \geq 2 m+3$ and $H^{1}(f ; \mathbb{Q})$ is injective then a model of the commutative CDGA square

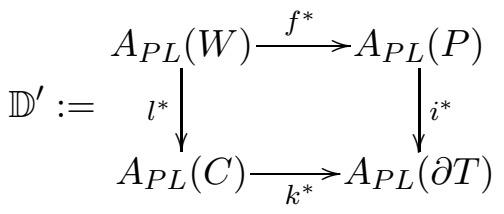

can be build explicitly out of any $C D G A$ model of $f^{*}: A_{P L}(W) \rightarrow A_{P L}(P)$.

More precisely, if $n \geq 2 m+4$ or if $n \geq 2 m+3$ and $H^{1}(f ; \mathbb{Q})$ is injective, then the commutative CDGA square $\mathbb{D}^{\prime}$ is weakly equivalent to any commutative CDGA square

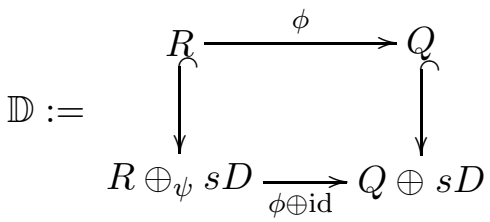

where

\footnotetext{
${ }^{2}$ It was called a shriek map in earlier versions of this paper.
} 
(i) $\phi: R \rightarrow Q$ is a CDGA model of $f^{*}: A_{P L}(W) \rightarrow A_{P L}(P)$ with $R^{>n}=0$ and $Q^{>m+2}=0$;

(ii) $D$ is a $Q$-DGmodule weakly equivalent to $s^{-n} \# Q$ with $D^{>n+1}=0$ and $D^{<n-m}=0$;

(iii) $\psi: D \rightarrow R$ is an $R$-DGmodules map such that $H^{n}(\psi)$ is an isomorphism and the mapping cones are endowed with the semi-trivial CDGA structure.

Moreover if $n \geq 2 m+3$ and $H^{1}(f ; \mathbb{Q})$ is injective, then $R, Q, D, \phi$, and $\psi$ satisfying (i)-(iii) can be explicitly constructed out of any CDGA model of $f^{*}: A_{P L}(W) \rightarrow A_{P L}(P)$.

Since CDGA models encode rational homotopy types of simply connected spaces an immediate corollary of the above theorem is that when $P$ and $W$ are simply connected and $\operatorname{dim}(W) \geq 2 \operatorname{dim}(P)+3$, then the rational homotopy type of the Poincaré embedding (1.1) depends only on the rational homotopy class of $f$.

As a byproduct of this theorem we obtain also a CDGA model $Q \oplus s s^{-n} \# Q$ of the boundary $\partial T$ of a thickening of $P$ under a high codimension hypothesis. This model was already described in [18 and an analogous model is built in [14] under weaker hypotheses.

In our first theorem we have supposed that $\operatorname{dim} W \geq 2 \operatorname{dim} P+3$. When the connectivity of the embedding is high this condition on the codimension can be weakened. Indeed in the case of PL-embeddings we have the following classical result:

Theorem 1.3 (PL-unknotting, Wall and Hudson) Let $P$ be a compact $m$ dimensional polyhedron and let $W$ be a closed $n$-dimensional manifold with $n \geq m+3$. Let $r$ be an integer such that

$$
r \geq 2 m-n+2
$$

Then any two homotopic $r$-connected embeddings $f_{0}, f_{1}: P \hookrightarrow W$ are isotopic. As a consequence, if $f$ is $r$-connected then the homotopy type of the square (1.1) depends only on the homotopy class of $f$.

Proof By the uniqueness part of the Wall's embedding theorem [29, page 76] $f_{0}$ and $f_{1}$ are concordant. Since the codimension is at least 3 , concordance implies isotopy [13]. Therefore $f_{0}$ is isotopic to $f_{1}$. By the uniqueness of a regular neighborhood this implies that the squares (1.1) for $f_{0}$ and $f_{1}$ are homeomorphic. 
The hypothesis that $f$ is $r$-connected with $r$ satisfying the inequality (1.2) is called the unknotting condition. The reason for which we have stated Theorem 1.3 in the context of PL-embeddings instead of Poincaré embeddings is that the corresponding result for Poincaré embeddings is known only under a slightly more restrictive condition. Indeed Klein has proved such an uniqueness result for Poincaré embeddings with an unknotting condition increased by one, i.e. $r \geq 2 m-n+3$ [15, Theorem 5.4], or with the sharp unknotting condition (1.2) in the metastable range [17. It is still an open question whether condition (1.2) guarantees the uniqueness of Poincaré embeddings in full generality.

We will prove a rational homotopy theoretical partial version of Theorem 1.3 by establishing that, under the unknotting condition (1.2), the rational homotopy type of the complement $C$ depends only on the rational homotopy class of $f$. From Theorem 1.2 a guess for the model of the complement would be $R \oplus_{\psi} s D$ with some assumption on the vanishing of $R, Q$, and $D$ in high degrees. This vanishing assumption can be removed if we truncate the mapping cone $R \oplus_{\psi} s D$ by a suitable acyclic module $L$. Moreover only a structure of $R$-DGmodule (instead of $Q$-DGmodule) is needed on $D$. More precisely we have the following theorem:

Theorem 1.4 Consider a Poincaré embedding (1.1) of codimension at least 2 with $P$ and $W$ connected. Let $r$ be a positive integer such that $H_{*}(f ; \mathbb{Q})$ is $r$-connected, that is $H_{i}(f ; \mathbb{Q})$ is an isomorphism for $i<r$ and an epimorphism for $i=r$.

If

$$
r \geq 2 m-n+2 \text {. }
$$

then a CDGA model of the map $l: C \rightarrow W$ can be build explicitly out of any $C D G A$ model of $f: P \rightarrow W$.

More precisely, let

(i) $\phi: R \rightarrow Q$ be a $C D G A$ model of $f^{*}: A_{P L}(W) \rightarrow A_{P L}(P)$ with $R$ connected;

(ii) $D$ be an $R$-DGmodule weakly equivalent to $s^{-n} \# Q$ with $D^{<n-m}=0$;

(iii) $\psi: D \rightarrow R$ be a top-degree map of $R$-DGmodules;

(iv) $L \subset R \oplus_{\psi} s D$ be an acyclic $R$-subDGmodule with $L^{\leq n-r-2}=0$ and $\left(R \oplus_{\psi} s D\right)^{\geq n-r} \subset L$.

Then the canonical CDGA map

$$
\lambda: R \rightarrow\left(R \oplus_{\psi} s D\right) / L
$$


is a CDGA-model of the map

$$
l^{*}: A_{P L}(W) \rightarrow A_{P L}(C) .
$$

where $\lambda$ is the composition of the inclusion with the projection and the algebra structure on the truncated mapping cone is induced by the formula $(r, s d)$. $\left(r^{\prime}, s d^{\prime}\right)=\left(r \cdot r^{\prime}, s\left(r \cdot d^{\prime} \pm r^{\prime} \cdot d\right)\right)$.

Moreover under condition (1.3) it is possible to construct explicitly $R, Q, D, L$, $\phi, \psi$ satisfying hypotheses (i)-(iv) out of any CDGA-model of $f^{*}: A_{P L}(W) \rightarrow$ $A_{P L}(P)$.

Corollary 1.5 Consider a Poincaré embedding (1.1) of codimension at least 3 and with $P$ and $W$ simply-connected. Let $r$ be a positive integer such that $H_{*}(f ; \mathbb{Q})$ is $r$-connected. If $r \geq 2 m-n+2$ then the rational homotopy type of the complement $C$ depends only on the rational homotopy class of $f$.

Moreover we will show that the unknotting condition in Theorem 1.4 is sharp. More precisely we will construct in Propositions 9.1 and 9.3 families of examples for which the unknotting condition (1.3) fails only by a little but such that the rational cohomology algebra of the complement is not determined by the rational homotopy class of the embedding. Note also that our rational result is valid for any Poincaré embeddings satisfying the unknotting condition, which improves by 1 the hypothesis under which the "integral" homotopy type of the complement is known to be unique [16, Corollary B].

Unfortunately we were not able to determine the complete rational homotopy type of the square (1.1) from the rational homotopy class of $f$ under the unknotting condition. The best result that we can prove in this direction is the determination, under connectivity hypotheses on $P$ and $W$ and the extra assumption that $n \geq m+r+2$, of the modified square (1.1) where $\partial T$ is replaced by the space $\partial \check{T}$ obtained by removing its top cell. See Theorem 8.2 for a precise statement.

Our rational models in Theorems 1.2 and 1.4 have applications to the construction of the model of blow-ups [21] and [19], and of the configuration space on two points [20].

The above discussion was about CDGA models for the square (1.1) which determine its rational homotopy type. Instead of CDGA models associated to the functor $A_{P L}$ we can associate models to the functor of singular cochains with coefficients in a field $\mathbf{k}$ of arbitrary characteristic, $S^{*}(-; \mathbf{k})$. If $Y$ is a space then $S^{*}(Y ; \mathbf{k})$ is a differential graded algebra (a DGA for short), and if 
$f: X \rightarrow Y$ is a continuous map then $S^{*}(X ; \mathbf{k})$ is a differential graded module (DGmodule) over the DGA $S^{*}(Y ; \mathbf{k})$. There is a notion of models of such DGmodules, and we can build such a model of the Poincaré embedding (1.1) without any restriction on the codimension or even on the connectivity of $P$. To state the result we use the notion of a menorah as defined in Example 3.2 and which is essentially a family of maps with same domain.

Theorem 1.6 Consider a Poincaré embedding (1.1) with $W$ connected. Denote the connected components of $P$ by $P_{1}, \cdots, P_{c}$ and set $f_{k}:=f \mid P_{k}$, for $k=1, \cdots, c$. Denote by $C^{*}$ one of the functors $S^{*}(-; \mathbf{k})$ or $A_{P L}$.

Suppose a quasi-isomorphism of DGA $\rho: A \stackrel{\simeq}{\rightarrow} C^{*}(W)$ has been given. Let

$$
\left\{\phi_{k}: R \rightarrow Q_{k}\right\}_{1 \leq k \leq c}
$$

be a model in $A$-DGMod of the menorah

$$
\left\{C^{*}\left(f_{k}\right): C^{*}(W) \rightarrow C^{*}\left(P_{k}\right)\right\}_{1 \leq k \leq c} .
$$

For $k=1, \cdots, c$, let $D_{k}$ be an $A$-DGmodule weakly equivalent to $s^{-n} \# C^{*}\left(P_{k}\right)$ and let $\psi_{k}: D_{k} \rightarrow R$ be a top-degree map of $A$-DGmodules.

Set $D=\oplus_{k=1}^{c} D_{k}, Q=\oplus_{k=1}^{c} Q_{k}, \phi=\left(\phi_{1}, \ldots, \phi_{c}\right): R \rightarrow Q$, and $\psi=$ $\sum_{k=1}^{c} \psi_{k}: D \rightarrow R$. Then the two following commutative squares are weakly equivalent in $A-D G M o d:$

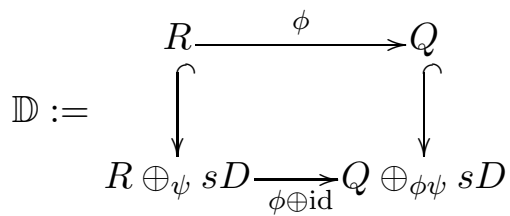

and

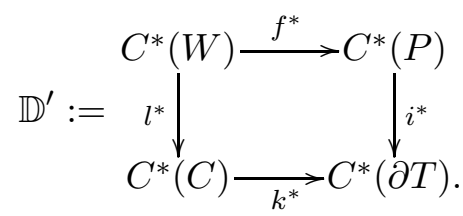

This DGmodule model enables us to improve the classical Lefschetz duality theorem. Indeed this classical result states that the cohomology of the complement, $H^{*}(C ; \mathbf{k})=H^{*}(W \backslash f(P) ; \mathbf{k})$, is determined as a vector space by the algebra map $H^{*}(f): H^{*}(W) \rightarrow H^{*}(P)$. Our result gives a way to determine the $H^{*}(W)$-module structure of $H^{*}(C)$, and even its algebra structure under the unknotting condition. This is the content of the following:

Corollary 1.7 (Improved Lefschetz duality) Consider a Poincaré embedding (1.1) with $W$ connected. Suppose a quasi-isomorphism of DGA $\rho: A \stackrel{\simeq}{\rightrightarrows}$ $C^{*}(W)$ has been given and let $\phi: R \rightarrow Q$ be an $A$-DGmodule model of $f^{*}: C^{*}(W) \rightarrow C^{*}(P)$. Then we have an isomorphism of $H^{*}(W ; \mathbf{k})$-modules

$$
H^{*}(C ; \mathbf{k}) \cong H\left(s^{-n} \# R \oplus_{s^{-n} \# \phi} s\left(s^{-n} \# Q\right)\right) \text {. }
$$


If moreover $H_{*}(f ; \mathbf{k})$ is $r$-connected with $r \geq 2 m-n+2$ then this isomorphism determines the algebra structure on $H^{*}(C ; \mathbf{k})$.

Examples of Section 9 will show that the unknotting condition cannot be dropped when determining the algebra structure in the last corollary. Christophe Boilley [1] has constructed examples showing that the $H^{*}(W)$ module structure on $H^{*}(C)$ is neither necessarily given by a trivial extension nor determined by the map $H^{*}(f)$ induced in cohomology.

Notice that in all the results of this paper we can replace the Poincaré embedding by the following weaker notion. Let $\mathbf{k}$ be a field. A $\mathbf{k}$-Poincaré embedding is a commutative square (1.1) such that $W,(P, \partial T)$ and $(C, \partial T)$ satisfy Poincaré duality in dimension $n$ over $\mathbf{k}, m$ is the cohomological dimension of $P$ with coefficients in $\mathbf{k}, H^{*}(i ; \mathbf{k})$ is $(n-m-1)$-connected, and the square (1.1) induces a Mayer-Vietoris long exact sequence in $H^{*}(-; \mathbf{k})$. In other words such a k-Poincaré embedding is a homological version of a Poincaré embedding.

As a last remark note that our study is complementary to the work of Morgan [25] who has computed the rational homotopy type of the complement of divisors $D_{i}$ with normal crossings in a projective algebraic variety $W$. In his case the codimension is very low ( $D_{i}$ is of codimension 2 ) but the existence of mixed Hodge structures [4 implies that the rational homotopy type of the complement is determined by the maps induced in cohomology by the inclusion of divisors. In the case of a single divisor $D$, Morgan's model for $W \backslash D$ is expressed in terms of the shriek map $f^{!}: H^{*+2}(D) \rightarrow H^{*}(W)$ which is a special case of our top-degree map (see Example 5.2.)

Plan of the rest of the paper Section 2 contains notation and terminology and Section 3 is about diagrams in closed model categories. We explain in this section what we mean by a model of a square or a menorah. In Section 4 we define the notion of a semi-trivial CDGA structure on certain mapping cones and in Section 5 we study the notion of a top-degree map and prove their existence and essential uniqueness. Section 6 is about the DGmodule model of a Poincaré embedding and contains the proofs of Theorem [1.6] and Corollary 1.7. Section 7 is about CDGA models of a Poincaré embedding in the stable case and contains the proof of Theorem 1.2. Section 8 discusses CDGA models of the complement in a Poincaré embedding under the unknotting condition. We prove here Theorem 1.4 and its corollaries. We also state and prove Theorem 8.2 which exhibits a model of a square related to (1.1) under a stronger unknotting condition. Finally Section 9 contains examples of rationally knotted embeddings and we illustrate by explicit examples the sharpness of the unknotting condition. 
Acknowledgements The authors want to thank Bill Dwyer for enlightening conversations on closed model structures on categories of diagrams and John Klein for explaining the proof of Theorem 1.3. We thank also the referee for pointing out that our results could apply to Poincaré embeddings. During this work the first author benefited from the hospitality of the University of Alberta and of a travel grant from F.N.R.S., and the second author from the hospitality of the Université of Louvain. The first author is Chercheur Qualifié au F.N.R.S.

\section{Notation and terminology}

We denote by $\mathbf{k}$ a commutative field. Recall the notions of differential graded algebra, or DGA for short, and of (left) graded differential modules over a DGA $R$, or $R$-DGmodules for short, as both defined for example in [8, Section 3(c)]. We will always suppose that the DGA are non negatively graded and that the differentials are of degree +1 . We denote by $R$-DGMod the category of $R$-DGmodules.

Convention on left and right modules Sometimes in the paper (in particular in Section (6) it will be important to distinguish between left and right DGmodules. By an $R$-DGmodule we always mean a left $R$-DGmodule, otherwise we write explicitly right $R$-DGmodule. Also by $R$-DGMod we denote only the category of left $R$-DGmodules. We denote by hom $($ resp. hom $R$ ) the sets of $\mathbf{k}$-modules (resp. $R$-modules) morphisms.

We have also a notion of commutative differential graded algebra, or CDGA for short, which is a DGA such that the multiplication is graded commutative (8. Example 5 in Section 3(b)] where there are called commutative cochain algebras). We denote by CDGA the corresponding category. A CDGA or more generally a non-negatively graded vector space, $V$, is called connected if $V^{0} \cong \mathbf{k}$.

The degrees of graded modules and algebras will be written as superscripts. If $X$ is a graded module or algebra, we will write $X^{>m}=0$ to express the fact that $X^{k}=0$ for $k>m$, and similarly $X^{\geq m}=0, X^{<m}=0$, and so on.

The dual of a graded $\mathbf{k}$-module $M$ will be denoted by \# $M$ with the grading $(\# M)^{i}=\operatorname{hom}\left(M^{-i}, \mathbf{k}\right)$. The duality pairing is defined by

$$
\langle-,-\rangle: M \otimes \# M \rightarrow \mathbf{k}, x \otimes f \mapsto\langle x, f\rangle=f(x) .
$$

If $(M, d)$ is a differential module then its dual \#M is equipped with the differential $\delta$ characterized by $\langle x, \delta(f)\rangle=-(-1)^{|x|}\langle d(x), f\rangle$. If $M$ is a right module 
over some graded algebra $R$, then its dual admits a structure of left $R$-module characterized by the formula $\langle x, a . f\rangle=\langle x . a, f\rangle$. Similarly if $M$ is a right DGmodule then its dual becomes a left DGmodule.

The $k$-th suspension of a graded vector space $M$ is the graded vector space $s^{k} M$ defined by $\left(s^{k} M\right)^{j} \cong M^{k+j}$ and this isomorphism is denoted by $s^{k}$. If $M$ is also a left $R$-module, we transport this structure on the $k$-suspension by the formula $r .\left(s^{k} x\right)=(-1)^{|r| k} s^{k}(r \cdot x)$. Also if $M$ is equipped with a differential $d$, then we define a differential on $s^{k} M$ by $d\left(s^{k} x\right)=(-1)^{k} s^{k}(d x)$. If $k=1$ we write $s M$ for $s^{1} M$.

The mapping cone of an $R$-DGmodule morphism $f: X \rightarrow Y$ is the $R$ DGmodule $C(f):=\left(Y \oplus_{f} s X, d\right)$ where the differential is defined by $d(y, s x)=$ $\left(d_{Y}(y)+f(x),-s d_{X}(x)\right)$. If $f$ is a CDGA morphism, in general there is no natural CDGA structure on the mapping cone but we will show in Section 4 that such a CDGA structure exists under favorable hypotheses.

We will use the functor of (normalized) singular cochains with coefficients in $\mathbf{k}$ $S^{*}(-; \mathbf{k})$ : Top $\rightarrow$ DGA as defined for example in [8, Chapter 5]. When $\mathbf{k}$ is of characteristic 0, we have also the de Rham-Sullivan functor of polynomial forms $A_{P L}:$ Top $\rightarrow$ CDGA as defined in [2] or [8, Chapter 10].

The categories $R$-DGMod and CDGA are closed model categories in the sense of Quillen for which the weak equivalences are the quasi-isomorphisms and the fibrations are the surjections (for a nice review of closed model categories, we refer the reader to [5]). By an acyclic (co)fibration we mean a (co)fibration that is also a weak equivalence. We say that two objects $X$ and $X^{\prime}$ in a closed model category are weakly equivalent or that $X$ is a model of $X^{\prime}$ if there exists a finite chain of weak equivalences joining them,

$$
X \stackrel{\simeq}{\longleftarrow} X_{1} \stackrel{\simeq}{\longrightarrow} \cdots X_{n} \stackrel{\simeq}{\longrightarrow} X^{\prime} .
$$

In that case we will write $X \simeq X^{\prime}$. Since in Section 3 we will consider a closed model structure on certain categories of diagrams, we can speak of models of that diagrams.

We review quickly the notion of relative Sullivan algebras which is an important class of cofibrations in CDGA. If $V$ is a non-negatively graded vector space we denote by $\wedge V$ the free graded commutative algebra generated by $V$ (see [8. $\S 3(\mathrm{~b})$, Example 6].) A relative Sullivan algebra ([8, Chapter 14], or $K S$ extension in the older terminology of [1]) is a CDGA morphism $\iota:\left(A, d_{A}\right) \hookrightarrow$ $(A \otimes \wedge V, D)$ where the differential $D$ is an extension of $d_{A}$ that satisfies some nilpotence condition (see [8, Chapter 14] for the precise definition.) Notice that 
in this paper we do not assume that $V^{0}=0$, following [11 but contrary to [8] In the special case $A=\mathbf{k}$ we get the notion of a Sullivan algebra, $(\wedge V, D)$, which is a cofibrant object in CDGA. Examples of cofibrant objects in $R$-DGMod are semi-free models as defined in [8, Chapter 6]. Roughly speaking they are $R$ DGmodules of the form $(R \otimes V, D)$ where $V$ is a graded vector space and the differential $D$ satisfies also a nilpotence condition. Finally remember that every object is fibrant in CDGA and in $R$-DGMod.

To denote that two maps $f_{0}$ and $f_{1}$ are homotopic in CDGA or $R$-DGMod we will write $f_{0} \sim f_{1}$, or sometimes $f_{0} \sim_{R} f_{1}$ to emphasize the underlying DGA. When $P$ and $N$ are $R$-DGmodules, with $P$ cofibrant, we denote by

$$
[P, N]_{R}
$$

the set of homotopy classes of $R$-DGmodules from $P$ to $N$.

\section{Diagrams in closed model categories}

In order of being able to speak of models of objects, maps, commutative squares, and so on, we review in this section the convenient language of diagrams as described for example in [5, Section 10]. There will exist a closed model structure on each of the categories of diagrams that we will consider. We will finish the section by two useful lemmas to turn certain homotopy commutative diagrams into commutative ones.

Definition 3.1 Let $\mathcal{S}$ be a small category and let $\mathcal{C}$ be any category. A diagram in $\mathcal{C}$ shaped on $\mathcal{S}$ is a covariant functor $\mathbb{D}: \mathcal{S} \rightarrow \mathcal{C}$ and we say that $\mathcal{S}$ is shaping the diagram. A morphism of diagrams is a natural transformation between two diagrams. This defines the category of diagrams $\mathcal{C}^{\mathcal{S}}$.

We describe now the five main examples of diagrams that we will consider in this paper. First recall that to each partially ordered set (or poset, for short), $(S, \leq)$, we can associate a small category $\mathcal{S}$ whose objects are the elements of $S$ and such that the set of morphisms, $\operatorname{hom}_{\mathcal{S}}(x, y)$, between two objects $x$ and $y$ in $\mathcal{S}$ is a singleton if $x \leq y$ and is the empty set otherwise.

\section{Examples 3.2}

Object If $\mathcal{S}$ is the category with only one object and one morphism (that is the category associated with the poset with only one element) then a diagram in $\mathcal{C}$ shaped on $\mathcal{S}$ is called an object of $\mathcal{C}$. 
Map If $\mathcal{S}$ is the category associated to the ordered set $\{0,1\}$ then a diagram in $\mathcal{C}$ shaped on $\mathcal{S}$ is just a map between two objects of $\mathcal{C}$. Such a diagram is called a map of $\mathcal{C}$.

Commutative square Let $\mathcal{S}$ be the category whose objects are the four sets $\emptyset,\{1\},\{2\}$, and $\{1,2\}$, and whose morphisms are the inclusion maps. A diagram in $\mathcal{C}$ shaped on $\mathcal{S}$ is called a commutative square in $\mathcal{C}$.

Menorah Let $\mathcal{S}$ be the category whose objects are $\emptyset,\{1\}, \cdots,\{n\}$, for some positive integer $n$ and where morphisms are inclusions of sets. Then a diagram in $\mathcal{C}$ shaped on $\mathcal{S}$ is just a collection of maps $f_{1}, \cdots, f_{n}$ with same domain. We call such a diagram a menorah and we denote it by $\left\{f_{i}\right\}_{1 \leq i \leq n}$.

Composite Let $\mathcal{S}$ be the category corresponding to the ordered set $\{0,1,2\}$. A diagram shaped on $\mathcal{S}$ is just two composable maps $f_{0}: X \rightarrow Y$ and $f_{1}: Y \rightarrow Z$. We call such a diagram a composite and we denote it by $\left(f_{0}, f_{1}\right)$.

Each category shaping one of the five diagrams in Example 3.2 is a very small category in the sense of [5, Section 10.13]. This notion is useful because of the following:

Proposition 3.3 Let $\mathcal{C}$ be a closed model category and let $\mathcal{S}$ be a very small category. Then the category $\mathcal{C}^{\mathcal{S}}$ of diagrams in $\mathcal{C}$ shaped on $\mathcal{S}$ admits a closed model structure such that a map $f: \mathbb{D} \rightarrow \mathbb{D}^{\prime}$ between diagrams is a weak equivalence (resp. a fibration) if and only if for each object $x$ in $\mathcal{S}$ the map $f(x): \mathbb{D}(x) \rightarrow \mathbb{D}^{\prime}(x)$ is a weak equivalence (resp. a fibration) in $\mathcal{C}$.

Moreover if $\hat{\mathbb{D}}$ is a cofibrant diagram in $\mathcal{C}^{\mathcal{S}}$ then for each object $x$ in $\mathcal{S}, \hat{\mathbb{D}}(x)$ is a cofibrant object of $\mathcal{C}$, and for each morphism $i$ in $\mathcal{S}$, the map $\hat{\mathbb{D}}(i)$ is a cofibration in $\mathcal{C}$.

If every object of $\mathcal{C}$ is fibrant, then the same is true in $\mathcal{C}^{\mathcal{S}}$.

Proof This model structure is described in [5, Section 10.13], where the cofibrations in $\mathcal{C}^{\mathcal{S}}$ are also defined (a complete proof of the axioms of Quillen for this category can be found in [10, Theorem 5.2.5]). Using the fact that the initial object $\emptyset$ in $\mathcal{C}^{\mathcal{S}}$ is the constant diagram with value $\emptyset$ at each object of $\mathcal{S}$, it is straightforward to check from the definition of a cofibration in $\mathcal{C}^{\mathcal{S}}(\underline{5}$, $10.13])$ that if $\emptyset \rightarrow \hat{\mathbb{D}}$ is a cofibration then each object $\hat{\mathbb{D}}(x)$ is cofibrant and each map $\hat{\mathbb{D}}(i)$ is a cofibration. The last statement is obvious. 
In this paper we will always suppose that the closed model structure on a category of diagrams $\mathcal{C}^{\mathcal{S}}$ is the one considered in Proposition 3.3. Following the terminology of Section 2 we can speak of weakly equivalent diagrams or of a model of a diagram.

Remark 3.4 If a menorah $\left\{f_{k}\right\}_{1 \leq k \leq n}$ is a model of another menorah $\left\{f_{k}^{\prime}\right\}_{1 \leq k \leq n}$, then clearly each map $f_{k}$ is a model of $f_{k}^{\prime}$. It is important to notice that the converse is not true in general. Similarly if a composite $(f, g)$ is a model of a composite $\left(f^{\prime}, g^{\prime}\right)$ then $f$ is a model of $f^{\prime}$ and $g$ is a model of $g^{\prime}$, but again the converse is not true.

The proofs of the following two lemmas are based on standard techniques of closed model categories and we leave them as exercises for the reader.

Lemma 3.5 Let $X$ and $X^{\prime}$ be two weakly equivalent objects in some closed model category in which every object is fibrant. Then there exists a cofibrant object $\hat{X}$ and acyclic fibrations

$$
X \underset{\beta}{\stackrel{\simeq}{\simeq}} \hat{X} \underset{\beta^{\prime}}{\stackrel{\simeq}{\longrightarrow}} X^{\prime}
$$

such that $\left(\beta, \beta^{\prime}\right): \hat{X} \rightarrow X \times X^{\prime}$ is also a fibration.

\section{Lemma 3.6 Let}

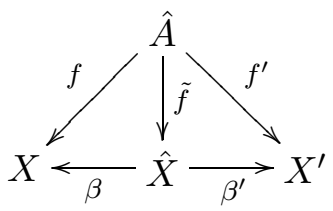

be a homotopy commutative diagram in a closed model category. If $\hat{A}$ is a cofibrant object, if $X$ and $X^{\prime}$ are fibrant, and if $\left(\beta, \beta^{\prime}\right): \hat{X} \rightarrow X \times X^{\prime}$ is a fibration then there exists a morphism $\hat{f}: \hat{A} \rightarrow \hat{X}$ homotopic to $\tilde{f}$ and making the following diagram strictly commute

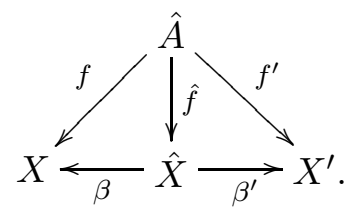




\section{CDGA structures on mapping cones}

The aim of this section is to define a natural extension of the $R$-DGmodule structure of some mapping cones to CDGA structures, under certain dimensionconnectivity hypotheses.

Definition 4.1 Let $R$ be a CDGA and let $f: X \rightarrow R$ be a morphism of $R$-DGmodules. Consider the mapping cone $C(f)=R \oplus_{f} s X$ and define a multiplication

$$
\mu: C(f) \otimes C(f) \rightarrow C(f)
$$

by, for homogeneous elements $r, r^{\prime} \in R$ and $x, x^{\prime} \in X$,

(i) $\mu\left(r \otimes r^{\prime}\right)=r \cdot r^{\prime}$

(ii) $\mu\left(r \otimes s x^{\prime}\right)=(-1)^{\operatorname{deg}(r)} s\left(r \cdot x^{\prime}\right)$

(iii) $\mu\left(s x \otimes r^{\prime}\right)=(-1)^{\operatorname{deg}(x) \cdot \operatorname{deg}\left(r^{\prime}\right)} s\left(r^{\prime} \cdot x\right)$

(iv) $\mu\left(s x \otimes s x^{\prime}\right)=0$.

This multiplication defines a commutative graded algebra structure (not necessarily differential) on $R \oplus_{f} s X$ that we call the semi-trivial CGA structure on the mapping cone.

This CGA structure on $C(f)$ is compatible with its $R$-module structure in the sense that the module structure is induced by the CGA map $R \hookrightarrow R \oplus_{f} s X$. It is important to notice that in general the multiplication $\mu$ defined above does not define a CDGA structure on $C(f)$ because the Leibnitz rule on the differential of the mapping cone is not necessarily satisfied. However, we have the following lemmas.

Lemma 4.2 Let $R$ be a CDGA and let $f: X \rightarrow R$ be an $R$-DGmodule morphism. Suppose that $(s X)^{<k}=0$ and $(R \oplus s X)^{>2 k}=0$ for some non negative integer $k$. Then the mapping cone $C(f)=R \oplus_{f} s X$ endowed with its semi-trivial multiplication is a CDGA and the inclusion map $R \hookrightarrow R \oplus_{f} s X$ is a CDGA-morphism.

Proof This lemma is a special case of the next lemma with $I=0$ and $l=$ 0 .

Lemma 4.3 Let $R$ be a CDGA, let $f: X \rightarrow R$ be an $R$-DGmodule morphism, and let $I \subset R \oplus_{f} s X$ be an $R$-DGsubmodule. Suppose that $(s X)^{<k}=0$, 
$I^{\leq k-l}=0$, and $\left(R \oplus_{f} s X\right)^{\geq 2 k-l+1} \subset I$ for non negative integers $k$ and $l$. Then the semi-trivial multiplication $\mu$ on the mapping cone $C(f)=R \oplus_{f} s X$ induces a multiplication on $C(f) / I$ which endows this quotient with a CDGA-structure, and the composition

$$
R \hookrightarrow R \oplus_{f} s X \stackrel{\mathrm{pr}}{\longrightarrow} C(f) / I
$$

is a CDGA morphism.

Proof We show first that $I$ is an ideal of the CGA $C(f)$ equipped with its semi-trivial CGA structure. Since $I$ is an $R$-submodule of $C(f)$ we have that $\mu(R \otimes I)=R . I \subset I$. On the other hand, for degree reasons $\mu(s X \otimes I) \subset$ $(C(f))^{\geq 2 k-l+1} \subset I$. Therefore $\mu(C(f) \otimes I) \subset I$. Thus $I$ is a left ideal, hence a two-sided ideal because $\mu$ is graded commutative.

This implies that the CGA structure on $C(f)$ induces a CGA structure on the quotient $C(f) / I$. Denote by $\delta$ the differential on the mapping cone $C(f)$ and by $\bar{\delta}$ the induced differential on the quotient. To prove that $(C(f) / I, \bar{\delta})$ is a CDGA we have only to check the Leibnitz formula. This will be a consequence of the following relation, for $c, c^{\prime}$ homogeneous elements in $R \oplus s X$ :

$$
\delta\left(\mu\left(c \otimes c^{\prime}\right)\right)-\mu\left(\delta(c) \otimes c^{\prime}\right)-(-1)^{|c|} \mu\left(c \otimes \delta\left(c^{\prime}\right)\right) \in I .
$$

To prove (4.1) we study different cases. If $c, c^{\prime} \in R$ then the expression in (4.11) is zero because $R$ is a DGA. If $c \in R$ and $c^{\prime} \in s X$ then the expression in (4.1) is zero because $\delta$ is a differential of $R$-DGmodule and the same is true if $c \in s X$ and $c^{\prime} \in R$ because $\mu$ is graded commutative. Finally if $c, c^{\prime} \in s X$ then the degree of the expression in (4.1) is at least $2 k+1 \geq 2 k-l+1$, therefore it belongs to $I$.

This completes the proof that $C(f) / I$ is a CDGA. It is straightforward to check that the map $R \rightarrow C(f) / I$ is a CDGA-morphism.

Definition 4.4 The CDGA-structures defined on the mapping cone $R \oplus_{f} s X$ in Lemma 4.2 (respectively on the truncated mapping cone $\left(R \oplus_{f} s X\right) / I$ in Lemma 4.3) is called the semi-trivial CDGA structure.

Our last lemma gives a sufficient condition for some DGmodule map between CDGA to be a CDGA morphism.

Lemma 4.5 Let $f: A \rightarrow B$ be a CDGA-morphism, let $A \stackrel{u}{\longrightarrow} A \otimes \wedge X$ be a relative Sullivan algebra, and let $\hat{f}: A \otimes \wedge X \rightarrow B$ be an $A$-DGmodule morphism extending $f$. If $X^{<k}=0$ and $B^{\geq 2 k}=0$ for some non negative integer $k$ then $\hat{f}$ is a CDGA morphism. 
Proof Since $A \otimes \wedge X$ and $B$ are graded commutative, $\hat{f}$ is a morphism of $A$-bimodules. The lemma follows from the fact that for degree reasons $\hat{f}(A \otimes$ $\left.\wedge^{\geq 2} X\right)=0$.

\section{Top-degree or shriek map}

The aim of this section is to introduce the simple notion of a top-degree map (which was called a shriek map in early version of this paper). A key result will be the existence and essential uniqueness of such top-degree maps (Proposition [5.6.)

We start with the definition and two examples.

Definition 5.1 Let $R$ be a DGA and assume that $H^{*}(R)$ is a connected Poincaré duality algebra in dimension $n$. A top-degree map of $R$-DGmodule is an $R$-DGmodule map $\psi: D \rightarrow R^{\prime}$ such that $R^{\prime}$ is weakly equivalent to $R$ and $H^{n}(\psi)$ is an isomorphism.

Example 5.2 Suppose that $f: V \hookrightarrow W$ is an embedding of connected closed oriented manifolds of codimension $k$. Denote by $[\mathrm{V}]$ and [W] their homology orientation classes. We have the classical cohomological shriek map (or Umkehr map, or Gysin map, see [3, VI.11.2])

$$
f^{!}: s^{-k} H^{*}(V ; \mathbf{k}) \rightarrow H^{*}(W ; \mathbf{k})
$$

characterized by the equation $f\left(s^{-k} v\right) \cap[W]=f_{*}(v \cap[V])$ (the $k$ th-suspension is here only to make $f^{!}$a degree preserving map.) It is clear that $f^{!}$is a map of $H^{*}(W)$-modules and that it induces an isomorphism in degree $n=\operatorname{dim}(W)$. Therefore $f^{!}$is a top-degree map of $H^{*}(W)$-module (here the differentials are supposed to be 0 ).

Example 5.3 Let $R$ be a DGA such that $H(R)$ is a connected Poincaré duality algebra in dimension $n$. Let $\phi: R \rightarrow Q$ be a morphism of right $R$-DGmodules such that $H^{0}(\phi)$ is an isomorphism. Then $s^{-n} \# R$ is quasiisomorphic to $R$ and the map

$$
s^{-n} \# \phi: s^{-n} \# Q \rightarrow s^{-n} \# R
$$

is a top-degree map of (left) $R$-DGmodules. 
To prove the existence and uniqueness of top-degree maps we need first to study further sets of homotopy classes of $R$-DGmodules. For an integer $i$, denote by $\operatorname{hom}_{R}^{i}(P, N)$ the $\mathbf{k}$-module of $R$-module maps of degree $i$ from $P$ to $N$ and set

$$
\operatorname{hom}_{R}^{*}(P, N):=\oplus_{i \in \mathbb{Z}} \operatorname{hom}_{R}^{i}(P, N) .
$$

We can define a degree +1 differential $\delta$ on this graded $\mathbf{k}$-module by the formula $\delta(f)=d_{N} f-(-1)^{|f|} f d_{P}$. The following identification is well-known and we omit its proof (e.g. [6]):

Lemma 5.4 Let $R$ be a DGA, let $P$ be a cofibrant $R$-DGmodule, and let $N$ be an $R$-DGmodule. Then we have an isomorphism

$$
[P, N]_{R} \cong H^{0}\left(\operatorname{hom}_{R}^{*}(P, N), \delta\right) \text {. }
$$

We have the following important characterization of the set of homotopy classes into a Poincaré duality algebra.

Proposition 5.5 Let $R$ be a DGA over a field $\mathbf{k}$ such that $H^{*}(R)$ is a connected Poincaré duality algebra in dimension $n$. Let $R^{\prime}$ be an $R$-DGmodule weakly equivalent to $R$ and let $P$ be a cofibrant $R$-DGmodule. Then the map

$$
H^{n}:\left[P, R^{\prime}\right]_{R} \rightarrow \operatorname{hom}_{\mathbf{k}}\left(H^{n}(P), H^{n}\left(R^{\prime}\right)\right), \quad[f] \mapsto H^{n}(f) .
$$

is an isomorphism of $\mathbf{k}$-modules.

Proof Without any loss of generality we can suppose that $R^{\prime}=R$ because weak equivalences preserve each side of the isomorphism we want to prove.

Since $H^{n}(R) \cong \mathbf{k}$ there exists a $\mathbf{k}$-DGmodule map $\epsilon_{0}: R \rightarrow s^{-n} \mathbf{k}$ inducing an isomorphism in $H^{n}$. Using the canonical isomorphism $\# s^{n} R \cong s^{-n} \# R$ we can interpret $\epsilon_{0}$ as a cocycle in $s^{-n} \# R$ and $\left[\epsilon_{0}\right] \neq 0$ in $H^{0}\left(s^{-n} \# R\right) \cong \# H^{n}(R)$. Since $R$ is also a right $R$-DGmodule, we have a structure of (left) $R$-DGmodule on $s^{-n} \# R$ (remember our convention in Section [2) There is a unique $R$ DGmodule map

$$
\epsilon: R \rightarrow s^{-n} \# R
$$

sending $1 \in R$ to $\epsilon_{0}$. Thus $H^{*}(\epsilon): H^{*}(R) \rightarrow s^{-n} \# H^{*}(R)$ is an $H^{*}(R)$-module morphism which is an isomorphism in degree $n$. By Poincaré duality of $H^{*}(R)$ this implies that $H^{*}(\epsilon)$ is an isomorphism in every degree. Thus $\epsilon$ is a quasiisomorphism.

Consider the adjunction isomorphism

$$
\operatorname{hom}_{R}(P, \# R) \cong \operatorname{hom}_{\mathbf{k}}(P, \mathbf{k}), \quad \phi \mapsto \hat{\phi}
$$


where $\hat{\phi}: P \rightarrow \mathbf{k}$ is defined by $\hat{\phi}(x)=(\phi(x))(1)$ for $x \in P$ and 1 the unit in $R$. Combining this isomorphism with Lemma 5.4 we get the following sequence of isomorphisms

$$
\begin{aligned}
{[P, R]_{R} } & \cong H^{0}\left(\operatorname{hom}_{R}(P, R)\right) \\
& \cong \epsilon^{0}\left(\operatorname{hom}_{R}\left(P, s^{-n} \# R\right)\right) \\
& \cong H^{n}\left(\operatorname{hom}_{R}(P, \# R)\right) \\
& \cong H^{n}\left(\operatorname{hom}_{\mathbf{k}}(P, \mathbf{k})\right) \\
& \cong \operatorname{hom}_{\mathbf{k}}\left(H^{n}(P), s^{n} \mathbf{k}\right)
\end{aligned}
$$

Moreover it is straightforward to check that the following diagram is commutative where the horizontal isomorphism is taken as the previous sequence of isomorphisms:

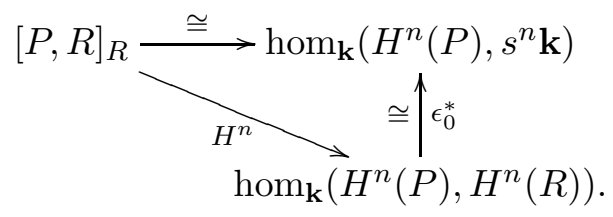

We establishes now the existence and uniqueness (up to homotopy and a scalar multiple) of top-degree maps.

Proposition 5.6 Let $R$ be a DGA such that $H^{*}(R)$ is a connected Poincaré duality algebra in dimension $n$, let $R^{\prime}$ be an $R$-DGmodule weakly equivalent to $R$, and let $\hat{D}$ be a cofibrant $R$-DGmodule such that $H^{n}(\hat{D}) \cong \mathbf{k}$. Then there exists a top-degree map of $R$-DGmodules

$$
\psi: \hat{D} \rightarrow R^{\prime} .
$$

Moreover if $\psi^{\prime}: \hat{D} \rightarrow R^{\prime}$ is another top-degree map then there exists $u \in \mathbf{k} \backslash\{0\}$ such that $[\psi]=u \cdot\left[\psi^{\prime}\right]$ in $\left[\hat{D}, R^{\prime}\right]_{R}$.

Proof By Proposition [5.5] we have an isomorphism

$$
H^{n}:\left[\hat{D}, R^{\prime}\right]_{R} \cong \operatorname{hom}_{\mathbf{k}}\left(H^{n}(\hat{D}), H^{n}\left(R^{\prime}\right)\right) .
$$

Denote by iso $\left(H^{n}(\hat{D}), H^{n}\left(R^{\prime}\right)\right)$ the submodule of $\operatorname{hom}_{\mathbf{k}}\left(H^{n}(\hat{D}), H^{n}\left(R^{\prime}\right)\right)$ consisting of isomorphisms. Since $H^{n}(\hat{D}) \cong \mathbf{k} \cong H^{n}(R)$ there is an obvious isomorphism

$$
\operatorname{iso}\left(H^{n}(\hat{D}), H^{n}\left(R^{\prime}\right)\right) \cong \mathbf{k} \backslash\{0\}
$$


Any homotopy class $\psi \in\left[\hat{D}, R^{\prime}\right]_{R}$ corresponding to an element of the non empty set iso $\left(H^{n}(\hat{D}), H^{n}\left(R^{\prime}\right)\right)$ gives a top-degree map, which proves the existence part.

The uniqueness part is based on the same computation and left to the reader.

We end this section by a lemma on sets of homotopy classes.

Lemma 5.7 Let $A$ be a DGA, let $D$ be a cofibrant $A$-DGmodule, and let $X$ be an $A$-DGmodule. Suppose that there exist integers $r \geq 1$ and $m \geq 0$ such that

- $H^{\leq r-1}(A)=H^{0}(A)=\mathbf{k}$, i.e. $A$ is cohomologically $(r-1)$-connected,

- $H^{<0}(X)=0$ and $H^{>m}(X)=0$, and

- $H \leq m-r+1(D)=0$.

Then the map

$$
H^{*}:[D, X]_{A} \rightarrow \operatorname{hom}_{\mathbf{k}}^{0}\left(H^{*}(D), H^{*}(X)\right), \quad[f] \mapsto H^{*}(f)
$$

is an isomorphism of $\mathbf{k}$-modules.

If moreover $r=1$ then $[D, X]_{A}=0$.

Proof We treat separately the cases $r=1$ and $r \geq 2$. Suppose first that $r=1$. Then $H^{\leq m}(D)=0=H^{>m}(X)$. By standard obstruction theory every $A$-DGmodule morphism $f: D \rightarrow X$ is nullhomotopic. Hence $[D, X]_{A}=0$. Moreover $\operatorname{hom}_{\mathbf{k}}^{0}\left(H^{*}(D), H^{*}(X)\right)=0$ for degree reasons. This proves the lemma for $r=1$.

Suppose that $r \geq 2$. Using Lemma 5.4 one can prove that the $\mathbf{k}$-module $[D, X]_{A}$ remains unchanged if we replace $D, X$, or $A$ by a cofibrant weakly equivalent objects (see [6, Proposition A.4.(ii)].) Since $H^{\leq 1}(A)=\mathbf{k}$, we can replace the DGA $A$ by a minimal free model in the sense of [12, Appendix], therefore we can suppose that $A^{\leq r-1}=\mathbf{k}$. Next by replacing $D$ by a weakly equivalent minimal semi-free $A$-DGmodule we can suppose that $D^{\leq m-r+1}=0$. Since $H^{>m}(X)=0$ and $A$ is connected we can also assume that $X^{>m}=0$.

Then, for degree reasons, the forgetful map $\phi^{i}: \operatorname{hom}_{A}^{i}(D, X) \rightarrow \operatorname{hom}_{\mathbf{k}}^{i}(D, X)$ is surjective for $i \geq-1$. Obviously $\phi^{i}$ is always injective. Thus in the following 
commutative diagram, the horizontal maps are isomorphisms:

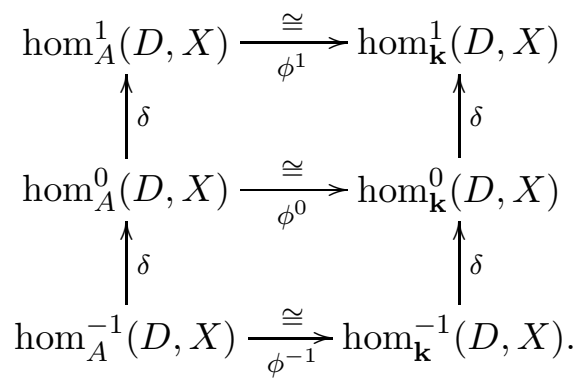

This implies that $H^{0}(\phi): H^{0}\left(\operatorname{hom}_{A}^{*}(D, X), \delta\right) \rightarrow H^{0}\left(\operatorname{hom}_{\mathbf{k}}^{*}(D, X), \delta\right)$ is an isomorphism. We conclude by using Lemma 5.4 and the obvious identification

$$
H^{0}\left(\operatorname{hom}_{\mathbf{k}}^{*}(D, X), \delta\right) \cong \operatorname{hom}_{\mathbf{k}}^{0}\left(H^{*}(D), H^{*}(X)\right)
$$

\section{DGmodule model of a Poincaré embedding}

The aim of this section is to prove Theorem 1.6 and Corollary 1.7

Remark 6.1 Before proceeding with the proof of Theorem 1.6 we make a comment about the hypothesis on the model of a menorah. Indeed in that theorem we suppose that $\left\{\phi_{k}\right\}_{1 \leq k \leq c}$ is a model of the menorah $\left\{C^{*}\left(f_{k}\right)\right\}_{1 \leq k \leq c}$. As we pointed out in Remark [3.4 when $c \geq 2$ this is a stronger hypothesis than asking for each $\phi_{k}$ to be a model of $C^{*}\left(f_{k}\right)$. We illustrate this fact by the following example. Consider the torus $T=S^{1} \times S^{1}$ and denote by $\dot{T}$ this torus with a small open disk removed, so that $\dot{T}$ is a compact surface of genus 1 with a circle for boundary. Let $f: S^{1} \hookrightarrow \dot{T}$ be an embedding such that composed with the inclusion $\dot{T} \subset S^{1} \times S^{1}$ it gives the inclusion of the first factor $S^{1}$ in $S^{1} \times S^{1}$. Denote by $\dot{T}_{1}$ and $\dot{T}_{2}$ two copies of $\dot{T}$ and let $f_{k}: S^{1} \hookrightarrow \dot{T}_{k}$ be the embeddings corresponding to $f, k=1,2$. Set $W=\dot{T}_{1} \cup_{\partial \dot{T}} \dot{T}_{2}$ which is a closed surface of genus 2. It is clear that the complement $C:=W \backslash\left(f_{1}\left(S^{1}\right) \amalg f_{2}\left(S^{1}\right)\right)$ is connected. Consider now the obvious automorphism $\phi$ of $W$ permuting $\dot{T}_{1}$ and $\dot{T}_{2}$. This automorphism is such that $\phi \circ f_{2}=f_{1}$. By deforming slightly $\phi$ into a diffeotopic automorphism $\phi^{\prime}$, we can suppose that $f_{2}^{\prime}:=\phi^{\prime} \circ f_{2}$ is an embedding of a circle closed but disjoint from $f_{1}\left(S^{1}\right)$. Then $C^{\prime}:=W \backslash\left(f_{1}\left(S^{1}\right) \amalg f_{2}^{\prime}\left(S^{1}\right)\right)$ is not connected. Thus $C^{*}(C)$ and $C^{*}\left(C^{\prime}\right)$ do not have the same DGmodule model since they have different cohomologies. On the other hand $C^{*}\left(f_{2}^{\prime}\right)$ and $C^{*}\left(f_{2}\right)$ do admit the same model since they differ only by the automorphism $C^{*}\left(\phi^{\prime}\right)$ of $C^{*}(W)$. The explanation of this apparent contradiction is in the fact 
that $\left\{C^{*}\left(f_{1}\right), C^{*}\left(f_{2}\right)\right\}$ and $\left\{C^{*}\left(f_{1}\right), C^{*}\left(f_{2}^{\prime}\right)\right\}$ do not admit a common model as menorah in the sense of Example 3.2 .

The proof of Theorem 1.6 consists of a series of four lemmas. Note first that by taking mapping cylinders we can assume without loss of generality that diagram (1.1) of Definition 1.1] is a genuine push-out and that each map $i, k, f, l$ is a closed cofibration.

Lemma 6.2 With the same hypotheses as in Theorem[1.6 consider the inclusion map $\iota: C^{*}(W, C) \rightarrow C^{*}(W)$. Then the commutative square $\mathbb{D}^{\prime}$ is weakly equivalent in $C^{*}(W)$-DGMod to the following commutative square:

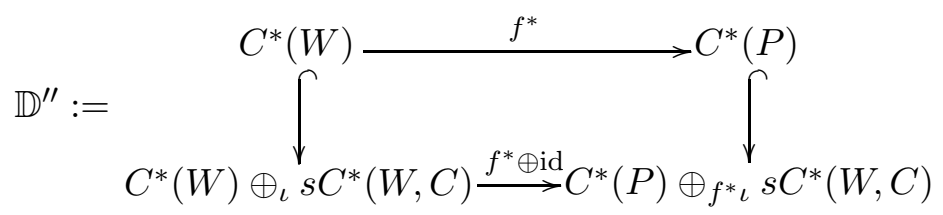

Proof Consider the following ladder of short exact sequences in $C^{*}(W)$ DGMod

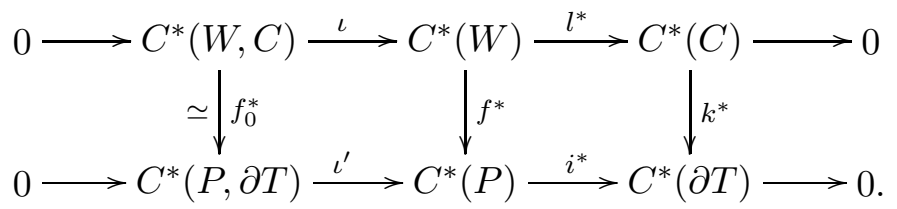

By Mayer-Vietoris $f_{0}^{*}$ is a quasi-isomorphism and we have a weak equivalence

$$
\operatorname{id} \oplus s f_{0}^{*}: \quad\left(C^{*}(P) \oplus_{\iota^{\prime} f_{0}^{*}} s C^{*}(W, C)\right) \stackrel{\simeq}{\rightrightarrows}\left(C^{*}(P) \oplus_{\iota^{\prime}} s C^{*}(P, \partial T)\right) .
$$

Thus in diagram $\mathbb{D}^{\prime \prime}$ we can replace the right bottom DGmodule by $C^{*}(P) \oplus_{\iota^{\prime}}$ $s C^{*}(P, \partial T)$. To finish the proof apply the five lemma to deduce that the map $k^{*}$ is weakly equivalent to the map induced between the mapping cones of $\iota$ and $\iota^{\prime}$.

Before stating the next two lemmas we need to introduce further notation. Let $\partial T_{k}$ be the union of the connected components of $\partial T$ that are sent to $P_{k}$ by $i$. Set $C_{k}:=C \cup_{\left(\partial T \backslash \partial T_{k}\right)}\left(P \backslash P_{k}\right)$, which can be interpreted as the complement of $P_{k}$ in $W$ since $W \simeq C_{k} \cup_{\partial T_{k}} P_{k}$. Define also the inclusion maps

$$
\iota_{k}: C^{*}\left(W, C_{k}\right) \hookrightarrow C^{*}(W) .
$$

In the next lemma we build a convenient common model $\hat{\phi}_{k}$ of both $\phi_{k}$ and $f_{k}^{*}$. 
Lemma 6.3 With the hypotheses of Theorem [1.6 there exists a cofibrant $A$-DGmodule $\hat{R}$, weak equivalences $\alpha, \alpha^{\prime}$, and, for each $k=1, \cdots, c$, an $A$ DGmodule cofibration $\hat{R} \stackrel{\hat{\phi}_{k}}{\longrightarrow} \hat{Q}_{k}$ and weak equivalences $\beta_{k}, \beta_{k}^{\prime}$, making the following diagrams commute

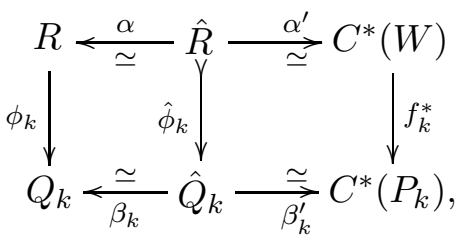

and such that $\left(\alpha, \alpha^{\prime}\right): \hat{R} \rightarrow R \oplus C^{*}(W)$ and $\left(\beta_{k}, \beta_{k}^{\prime}\right): \hat{Q}_{k} \rightarrow Q_{k} \oplus C^{*}\left(P_{k}\right)$ are surjective.

Proof Let $\mathcal{S}$ be the category shaping menorah's. Apply Lemma 3.5 in the category $A$-DGMod ${ }^{\mathcal{S}}$ to get a cofibrant menorah $\left\{\hat{\phi}_{k}\right\}_{1 \leq k \leq c}$ and weak equivalences

$$
\left\{\phi_{k}\right\}_{1 \leq k \leq q} \underset{\left.\left(\alpha, \beta_{k}\right)\right\}_{k}}{\stackrel{\simeq}{\simeq}}\left\{\hat{\phi}_{k}\right\}_{1 \leq k \leq c} \underset{\left\{\left(\alpha^{\prime}, \beta_{k}^{\prime}\right)\right.}{\simeq}\left\{f_{k}^{*}\right\}_{1 \leq k \leq c}
$$

with the desired properties. In particular by the second part of Proposition 3.3 the maps $\hat{\phi}_{k}: \hat{R} \rightarrow \hat{Q}_{k}$ are cofibrations between cofibrant objects.

Lemma 6.4 With the hypotheses of Theorem [1.6 and with the notation of Lemma 6.3, there exist for each $k=1, \cdots, c$, a cofibrant $A$-DGmodule, $\hat{D}_{k}$, and weak equivalences of $A$-DGmodules,

$$
D_{k} \stackrel{\gamma_{k}}{\simeq} \hat{D}_{k} \stackrel{\gamma_{k}^{\prime}}{\simeq} C^{*}\left(W, C_{k}\right),
$$

making the following diagram of isomorphisms commute

$$
\begin{gathered}
H^{n}\left(D_{k}\right) \stackrel{H^{n}\left(\gamma_{k}\right)}{\cong} H^{n}\left(\hat{D}_{k}\right) \stackrel{H^{n}\left(\gamma_{k}^{\prime}\right)}{\cong} H^{n}\left(W, C_{k}\right) \\
H^{n}\left(\psi_{k}\right) \downarrow \cong \\
H^{n}(R) \underset{H^{n}(\alpha)}{\cong} H^{n}(\hat{R}) \frac{\coprod}{H^{n}\left(\alpha^{\prime}\right)} H^{n}(W) .
\end{gathered}
$$

Proof Fix $k=1, \cdots, c$. By hypothesis $D_{k}$ is weakly equivalent as an $A$ DGmodule to $s^{-n} \# C^{*}\left(P_{k}\right)$, by Poincaré duality to $C^{*}\left(P_{k}, \partial T_{k}\right)$, and by MayerVietoris to $C^{*}\left(W, C_{k}\right)$. By Lemma 3.5. we can find a cofibrant $A$-DGmodule, $\hat{D}_{k}$, and weak equivalences of $A$-DGmodules

$$
D_{k} \stackrel{\gamma_{k}}{\simeq} \hat{D}_{k} \stackrel{\gamma_{k}^{\prime \prime}}{\simeq} C^{*}\left(W, C_{k}\right) \text {. }
$$


By Lefschetz duality $H^{n}\left(W, C_{k}\right) \cong H_{0}\left(P_{k}\right) \cong \mathbf{k}$ and $H^{n}\left(\iota_{k}\right)$ is an isomorphism. By definition of a top-degree map $H^{n}\left(\psi_{k}\right)$ is also an isomorphism. Thus the diagram appearing in the statement of the lemma, with $\gamma_{k}^{\prime \prime}$ replacing $\gamma_{k}^{\prime}$, is indeed a diagram of isomorphisms. Since $H^{n}\left(\hat{D}_{k}\right) \cong H^{n}(\hat{R}) \cong \mathbf{k}$, the two isomorphisms

$$
H^{n}(\alpha)^{-1} H^{n}\left(\psi_{k}\right) H^{n}\left(\gamma_{k}\right) \text { and } H^{n}\left(\alpha^{\prime}\right)^{-1} H^{n}\left(\iota_{k}\right) H^{n}\left(\gamma_{k}^{\prime \prime}\right)
$$

differ only by a multiplicative constant $u \in \mathbf{k} \backslash\{0\}$. Set $\gamma_{k}^{\prime}:=u \cdot \gamma_{k}^{\prime \prime}$ which is also a weak equivalence of $A$-DGmodules. Then the diagram of isomorphisms of the statement commutes.

Recall the notion of model of a composite from Example 3.2

Lemma 6.5 With the hypotheses of Theorem 1.6, the composite

$$
D \stackrel{\psi}{\longrightarrow} R \stackrel{\phi}{\longrightarrow} Q
$$

is an A-DGmodule model of the composite

$$
C^{*}(W, C) \stackrel{\iota}{\longrightarrow} C^{*}(W) \stackrel{f^{*}}{\longrightarrow} C^{*}(P) .
$$

Proof Consider all the morphisms and DGmodules built in Lemma 6.3 and Lemma 6.4 Fix $k=1, \cdots, c$. Take a lifting of $A$-DGmodules $\hat{\psi}_{k}: \hat{D}_{k} \rightarrow \hat{R}$ of $\psi_{k} \gamma_{k}$ along the acyclic fibration $\alpha$, so that

$$
\alpha \hat{\psi_{k}}=\psi_{k} \gamma_{k}
$$

which is a top-degree map. Also $\alpha^{\prime} \hat{\psi}_{k}$ and $\iota_{k} \gamma_{k}^{\prime}$ are top-degree maps with values in $C^{*}(W)$. By Proposition 5.6 there are homotopic up to a multiplicative scalar $u \neq 0$ and Lemma 6.4 and (6.1) imply that $u=1$. Thus

$$
\alpha^{\prime} \hat{\psi}_{k} \simeq_{A} \iota_{k} \gamma_{k}^{\prime} \text {. }
$$

Set $\hat{D}:=\oplus_{k=1}^{c} \hat{D}_{k}, \quad \gamma:=\oplus_{k=1}^{c} \gamma_{k}, \gamma^{\prime}:=\oplus_{k=1}^{c} \gamma_{k}^{\prime}$, and $\hat{\psi}:=\sum_{k=1}^{c} \hat{\psi}_{k}$. Since the $P_{k}$ 's are pairwise disjoint, we have an identification $C^{*}(W, C)=$ $\oplus_{k=1}^{c} C^{*}\left(W, C_{k}\right)$. Equations (6.1) and (6.2) yield to the following homotopy commutative diagram in $A$-DGMod

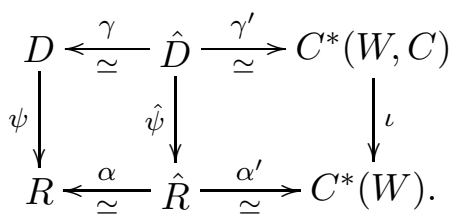


Since $\left(\alpha, \alpha^{\prime}\right)$ is a fibration we can suppose by Lemma 3.6 that $\hat{\psi}$ has been chosen such that the above diagram is strictly commutative. Gluing this diagram with that built in Lemma 6.3 we get a commutative diagram of $A$-DGmodules

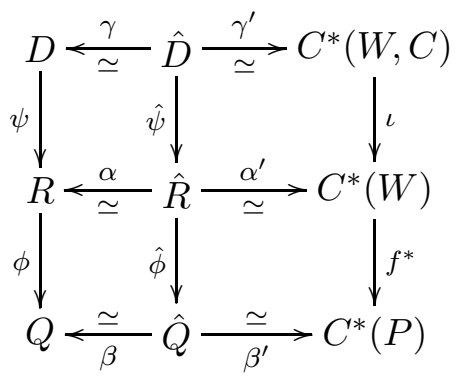

and the lemma is proved.

Collecting the four previous lemmas we achieve the proof of Theorem [1.6] and its corollary.

Proof of Theorem 1.6 Recall diagrams $\mathbb{D}$ and $\mathbb{D}^{\prime}$ defined in Theorem 1.6 and diagram $\mathbb{D}^{\prime \prime}$ defined in Lemma 6.2 Using Lemma 6.5 and taking mapping cones we deduce that the diagrams $\mathbb{D}$ and $\mathbb{D}^{\prime \prime}$ are weakly equivalent in $A$ DGMod. By Lemma 6.2 diagrams $\mathbb{D}^{\prime}$ and $\mathbb{D}^{\prime \prime}$ are also weakly equivalent in $A$-DGMod.

Proof of Corollary 1.7 By Example 5.3 $s^{-n} \# \phi$ is a top-degree map of right $A$-DGmodules. Theorem 1.6 implies that $s^{-n} \# R \oplus_{s^{-n} \# \phi} s s^{-n} \# Q$ is a right $A$-DGmodule model of $C^{*}(C)$. Therefore their homologies are isomorphic as right $H^{*}(W)$-modules and by commutativity also as left modules.

Since $H^{>m}(P)=0$ and by Lefschetz duality, $H_{<n-m}(W, C)=0$ and $H^{i}(W) \rightarrow$ $H^{i}(C)$ is an isomorphism for $i<n-m-1$. Therefore if $x . y$ is a product in $H^{*}(C)$ that is not determined by the $H^{*}(W)$-module structure then $\operatorname{deg}(x), \operatorname{deg}(y) \geq n-m-1$. Hence $\operatorname{deg}(x . y) \geq 2(n-m-1) \geq n-r$. Since $H^{*}(f)$ is $r$-connected we have that $H_{\leq r}(W, P)=0$ and by Lefschetz duality $H^{\geq n-r}(C)=0$. Therefore $x . y=0$.

\section{CDGA model of a Poincaré embedding in the sta- ble case}

In this section we give a proof of Theorem 1.2. Here is an overview of that proof. 
(1) We want to show that the diagrams $\mathbb{D}$ and $\mathbb{D}^{\prime}$ are weakly equivalent as commutative squares of CDGA. By Theorem [1.6 we already know that they are weakly equivalent in a certain category of DGmodules.

(2) We will build a convenient common CDGA model $\hat{R} \stackrel{\hat{\phi}}{\longrightarrow} \hat{Q}$ of both $\phi: R \rightarrow Q$ and $f^{*}: A_{P L}(W) \rightarrow A_{P L}(P)$. We can then consider the category of " $\hat{\phi}$-DGmodules" whose objects consist of maps of $\hat{R}$-DGmodules $M \rightarrow N$ such that $N$ is also equipped with a $\hat{Q}$-DGmodule compatible with its $\hat{R}$-DGmodule structure through the map $\hat{\phi}$. The morphisms of this category consist of certain commutative squares that we call $\hat{\phi}$ squares (see Definition (7.7). In particular the diagrams $\mathbb{D}$ and $\mathbb{D}^{\prime}$ will be $\hat{\phi}$-squares.

(3) A refinement of the arguments of Theorem [1.6 will show that the diagrams $\mathbb{D}$ and $\mathbb{D}^{\prime}$ are weakly equivalent not only as squares in the category of $\hat{R}$-DGmodules but also as $\hat{\phi}$-squares, which means that the weak equivalences between the right sides of diagrams $\mathbb{D}$ and $\mathbb{D}^{\prime}$ will be of $\hat{Q}$-DGmodules (Lemma 7.9])

(4) Using the results of Section 4 (notably Lemma 4.5), we will show that this weak equivalence of $\hat{\phi}$-squares between $\mathbb{D}$ and $\mathbb{D}^{\prime}$ is indeed a weak equivalence of CDGA squares.

Let's move to the details by establishing a series of lemmas. Note first that without loss of generality we can assume that (1.1) is a genuine push-out and that $f$ induces a map of pairs $f_{0}:(P, \partial T) \rightarrow(W, C)$. In the next lemma we build a common model $\hat{R} \stackrel{\hat{\phi}}{\longrightarrow} \hat{Q}$ of both $\phi$ and $f^{*}$.

Lemma 7.1 With the hypotheses of Theorem 1.2 there exists a cofibrant CDGA $\hat{R}$, a relative Sullivan algebra $\hat{R} \stackrel{\hat{\phi}}{\longrightarrow} \hat{Q}$, and a commutative diagram of CDGA where horizontal arrows are weak equivalences

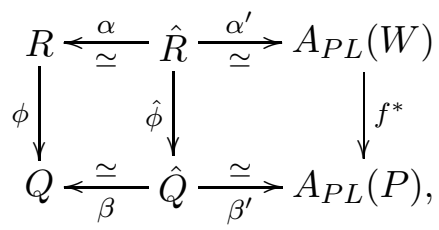

and $\left(\alpha, \alpha^{\prime}\right): \hat{R} \rightarrow R \oplus A_{P L}(W)$ and $\left(\beta, \beta^{\prime}\right): \hat{Q} \rightarrow Q \oplus A_{P L}(P)$ are surjections.

Proof This is a consequence of Lemma 3.5 in the the category of maps in CDGA, of the second part of Proposition 3.3, and of the fact that every CDGA 
cofibration is a retract of a Sullivan relative algebra. Alternatively the lemma can be proved using standard techniques of 8 .

Our next lemma gives a replacement $\bar{R}$ of $\hat{R}$ that fibres on different DGmodules.

Lemma 7.2 With the hypotheses of Theorem 1.2 and the notation of Lemma 7.1 there exists a factorization of $\hat{R}$-DGmodules of $\left(\alpha, \alpha^{\prime}, \hat{\phi}\right)$ into an acyclic cofibration $\rho$ followed by a fibration $\left(\bar{\alpha}, \bar{\alpha}^{\prime}, \bar{\phi}\right)$ as follows:

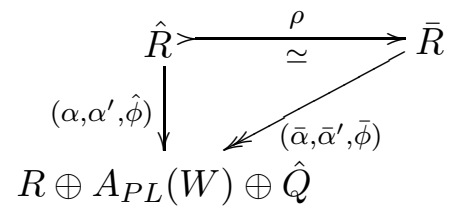

Proof The existence of such a factorization is one of the axioms of the closed model structure on the category $\hat{R}$-DGMod.

In the following lemma we give a common model $\hat{D}$ of both $D$ and $A_{P L}(P, \partial T)$.

Lemma 7.3 With the hypotheses of Theorem 1.2 and with the notation of Lemmas 7.1] and 7.2, there exists a cofibrant $\hat{Q}$-DGmodule, $\hat{D}$, and weak equivalences of $\hat{Q}-D G m o d u l e s$,

$$
D \stackrel{\gamma}{\simeq} \hat{D} \stackrel{\gamma^{\prime}}{\simeq} A_{P L}(P, \partial T),
$$

making the following diagram of isomorphisms commute

$$
\begin{gathered}
H^{n}(D) \stackrel{H^{n}(\gamma)}{\cong} H^{n}(\hat{D}) \stackrel{H^{n}\left(\gamma^{\prime}\right)}{\cong} H^{n}(P, \partial T) \stackrel{H^{n}\left(f_{0}\right)}{\cong} H^{n}(W, C) \\
H^{n}(\psi) \mid \cong \\
H^{n}(R) \underset{H^{n}(\iota)}{\stackrel{\cong}{H^{n}(\bar{\alpha})} H^{n}(\bar{R}) \frac{\cong}{H^{n}\left(\bar{\alpha}^{\prime}\right)} H^{n}(W) .}
\end{gathered}
$$

Moreover $\hat{D}$ is also a cofibrant $\hat{R}$-DGmodule and there exists an $\hat{R}$-DGmodule weak equivalence

$$
\gamma^{\prime \prime}: \hat{D} \stackrel{\cong}{\rightrightarrows} A_{P L}(W, C)
$$

making the following diagram commute

$$
\begin{aligned}
& \begin{array}{r}
\hat{D} \underset{\gamma^{\prime}}{\stackrel{\gamma^{\prime \prime}}{\longrightarrow}} A_{P L}(W, C) \\
\simeq \simeq f_{0}^{*}
\end{array} \\
& A_{P L}(P, \partial T) \text {. }
\end{aligned}
$$


Proof The proof of the first part of the lemma is similar to the proof of Lemma 6.4 .

For the second part of the lemma, note that by [8, Lemma 14.1] $\hat{D}$ is a cofibrant $\hat{R}$-DGmodule because it is a cofibrant $\hat{Q}$-DGmodule and because $\hat{\phi}: \hat{R} \rightarrow \hat{Q}$ is a relative Sullivan algebra. Also $f_{0}^{*}$ is a surjective quasi-isomorphism. We take $\gamma^{\prime \prime}$ as a lift of $\gamma^{\prime}$ along the acyclic fibration $f_{0}^{*}$.

Lemma 7.4 With the hypotheses of Theorem 1.2 and with the notation of Lemmas 7.1] 7.3, there exists an $\hat{R}$-DGmodule morphism

$$
\tilde{\psi}: \hat{D} \rightarrow \bar{R}
$$

making the following diagram homotopy commute in $\hat{R}$-DGMod

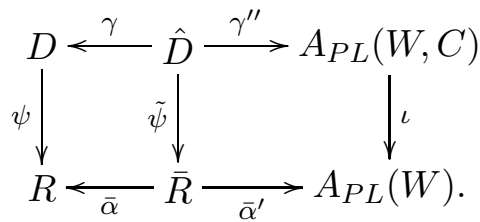

Proof The argument is the same as in the beginning of the proof of Lemma 6.5 .

We build now a $\hat{Q}$-DGmodule common model $\chi$ both of $\phi \psi=0$ and of $\iota^{\prime}: A_{P L}(P, \partial T) \rightarrow A_{P L}(P)$.

Lemma 7.5 With the hypotheses of Theorem 1.2 and with the notation of Lemmas 7.1 7.4 the composite $\phi \psi$ is a $Q$-DGmodule morphism and there exists a $\hat{Q}$-DGmodule morphism

$$
\chi: \hat{D} \rightarrow \hat{Q}
$$

making the following diagram commute in $\hat{Q}-D G M o d$

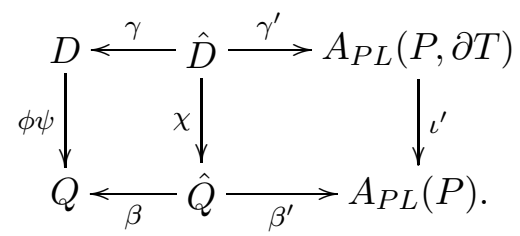

Moreover $\chi \simeq_{\hat{R}} \bar{\phi} \tilde{\psi}$. 
Proof Notice that for degree reasons $\phi \psi=0$, therefore it is a morphism of $Q$-DGmodules. Applying Lemma [5.7 with $r=1$ we get that $[\hat{D}, Q]_{\hat{Q}}=0=$ $\left[\hat{D}, A_{P L}(T)\right]_{\hat{Q}}$. Therefore the diagram of the statement with 0 replacing $\chi$ is homotopy commutative in $\hat{Q}$-DGMod. Since $\left(\beta, \beta^{\prime}\right)$ is a fibration, Lemma 3.6 permits to replace the zero map by a homotopic $\hat{Q}$-DGmodule morphism $\chi$ making the diagram strictly commute.

We have also by Lemma 5.7 that $[\hat{D}, \hat{Q}]_{\hat{R}}=0$, hence $\chi \simeq_{\hat{R}} \bar{\phi} \tilde{\psi}$.

Lemma 7.6 With the hypotheses of Theorem 1.2 and with the notation of Lemmas 7.1 7.5, there exists a morphism of $\hat{R}$-DGmodule $\bar{\psi}: \hat{D} \rightarrow \bar{R}$ making both of the following diagrams commute

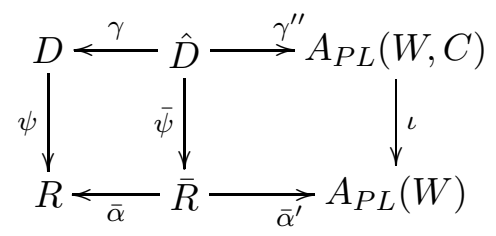

and

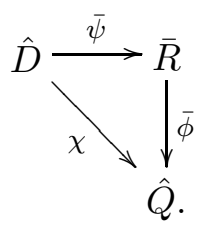

Proof By Lemmas 7.4 and 7.5 , we have the following homotopy commutative diagram in $\hat{R}$-DGMod

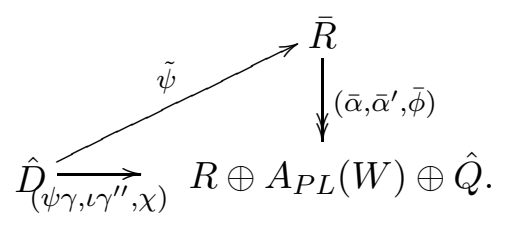

Since $\left(\bar{\alpha}, \bar{\alpha}^{\prime}, \bar{\phi}\right)$ is a fibration and $\hat{D}$ is cofibrant, a standard argument in closed model categories shows that we can replace $\tilde{\psi}$ by a homotopic map $\bar{\psi}$ making the diagram strictly commute.

As we have explained in the overview of the proof, in order to prove that diagrams $\mathbb{D}$ and $\mathbb{D}^{\prime}$ of Theorem 1.2 are weakly equivalent in CDGA, we will first prove that there are weakly equivalent as " $\hat{\phi}$-squares" that we define now. To give a meaning to this assertion we could define a genuine closed model structure on the category of $\hat{\phi}$-squares. Instead of doing so we prefer to introduce the following ad hoc definition of weakly equivalent $\hat{\phi}$-squares.

Definition 7.7 Let $\hat{\phi}: \hat{R} \rightarrow \hat{Q}$ be a CDGA morphism. 
(i) By a $\hat{\phi}$-square we mean a commutative square of $\hat{R}$-DGmodules

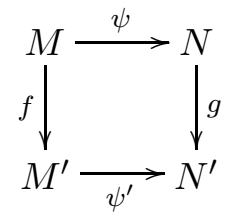

such that $N$ and $N^{\prime}$ have also a structure of $\hat{Q}$-DGmodule compatible with their $\hat{R}$-DGmodule structure through $\hat{\phi}$ and such that the right map $g$ is a $\hat{Q}$-DGmorphism.

(ii) A morphism of $\hat{\phi}$-squares is a morphism, $\Theta$, of commutative squares in $\hat{R}$-DGmodules between two $\hat{\phi}$-squares

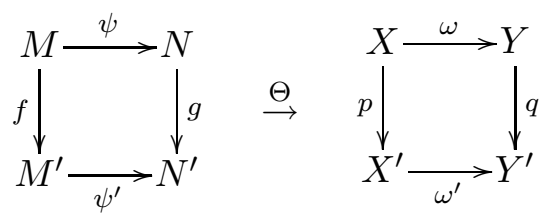

of the form $\Theta=\left(\begin{array}{cc}\mu & \nu \\ \mu^{\prime} & \nu^{\prime}\end{array}\right)$ such that $\nu$ and $\nu^{\prime}$ are also morphisms of $\hat{Q}$-DGmodules.

(iii) A morphism $\Theta$ of $\hat{\phi}$-squares is called a fibration (resp. a weak equivalence) if each of the morphisms $\mu, \mu^{\prime}, \nu, \nu^{\prime}$ is a surjection (resp. quasiisomorphism). A morphism of $\hat{\phi}$-squares which is both a fibration and a weak equivalence is called an acyclic fibration.

Recall the diagrams $\mathbb{D}$ and $\mathbb{D}^{\prime}$ from the statement of Theorem 1.2

Lemma 7.8 With the hypotheses of Theorem 1.2 and with the notation of Lemmas 7.1 7.6. Diagrams $\mathbb{D}$ and $\mathbb{D}^{\prime}$ are both commutative squares in CDGA and $\hat{\phi}$-squares. The following diagram

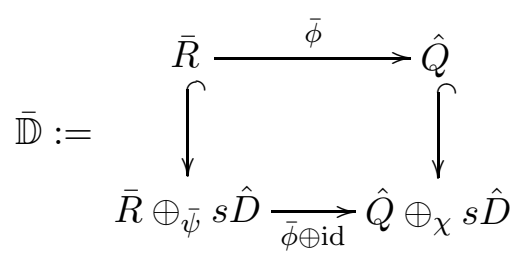

is a $\hat{\phi}$-square. 
Proof The CDGA structure on the mapping cones of the bottom side of Diagram $\mathbb{D}$ are the semi-trivial CDGA structures, which exist by Lemma 4.2 . From this it is clear that $\mathbb{D}$ is a commutative square of CDGA, as well as $\mathbb{D}^{\prime}$. They are also $\hat{\phi}$-squares with $\hat{R}$ - and $\hat{Q}$-DGmodule structures induced by the maps $\alpha, \alpha^{\prime}, \beta$, and $\beta^{\prime}$.

Using the fact that $\chi$ is a $\hat{Q}$-DGmodule morphism it is immediate to check that $\overline{\mathbb{D}}$ is a $\hat{\phi}$-square.

Lemma 7.9 With the hypotheses of Theorem 1.2 and with the notation of Lemmas 7.1 7.8, there exist acyclic fibrations of $\hat{\phi}$-squares

$$
\mathbb{D} \underset{\simeq}{\stackrel{\Theta}{\simeq}} \stackrel{\Theta^{\prime}}{\simeq} \mathbb{D}^{\prime}
$$

Proof Using the different maps constructed in our previous series of lemmas we will describe these two acyclic fibrations explicitly. Consider the following commutative square

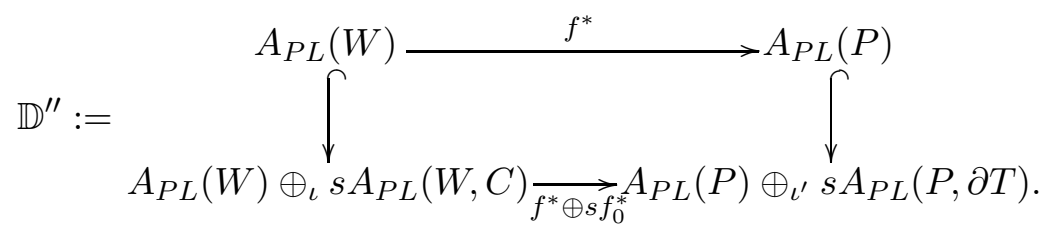

Using the fact that $\iota^{\prime}: A_{P L}(P, \partial T) \rightarrow A_{P L}(P)$ is a morphism of $A_{P L}(P)$ DGmodules, hence of $\hat{Q}$-DGmodules, we see that $\mathbb{D}^{\prime \prime}$ is a diagram of $\hat{\phi}$-squares. Clearly $\Theta^{\prime \prime \prime}:=\left(\begin{array}{cc}\text { id } & \text { id } \\ l^{*} \oplus 0 & i^{*} \oplus 0\end{array}\right): \mathbb{D}^{\prime \prime} \rightarrow \mathbb{D}^{\prime}$ is a surjection, and an argument analogous to that of Lemma 6.2 shows that it is a weak equivalence. Hence $\Theta^{\prime \prime \prime}$ is an acyclic fibration. We have another acyclic fibration $\Theta^{\prime \prime}: \overline{\mathbb{D}} \simeq \mathbb{D}^{\prime \prime}$ given by $\Theta^{\prime \prime}:=\left(\begin{array}{cc}\bar{\alpha}^{\prime} & \beta^{\prime} \\ \bar{\alpha}^{\prime} \oplus s \gamma^{\prime \prime} & \beta^{\prime} \oplus s \gamma^{\prime}\end{array}\right)$. Then $\Theta^{\prime}:=\Theta^{\prime \prime \prime} \Theta^{\prime \prime}$ is one of the required acyclic fibration. The other one is given by $\Theta:=\left(\begin{array}{cc}\bar{\alpha} & \beta \\ \bar{\alpha} \oplus s \gamma & \beta \oplus s \gamma\end{array}\right)$.

We sketch now an overview of the end of the proof of the Theorem. In the next lemma we build an intermediate commutative square, $\hat{\mathbb{D}}$, which is a CDGA model of $\mathbb{D}^{\prime}$. Moreover $\hat{\mathbb{D}}$ is also a "cofibrant $\hat{\phi}$-square", therefore by lifting along the quasi-isomorphisms $\Theta$ and $\Theta^{\prime}$ we will deduce that $\hat{\mathbb{D}}$ is a model of $\hat{\phi}$-square of $\mathbb{D}$. Finally a degree argument will imply that this $\hat{\phi}$-square quasiisomorphism $\hat{\Theta}: \hat{\mathbb{D}} \simeq \mathbb{D}$ is in fact of CDGA and this will prove that $\mathbb{D}$ and $\mathbb{D}^{\prime}$ are weakly equivalent CDGA squares. Let's move to the details. 
Lemma 7.10 With the hypotheses of Theorem 1.2 and with the notation of Lemmas 7.1 and 7.8, there exists a commutative square in CDGA,

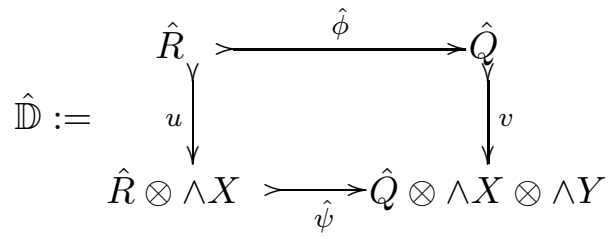

where $\hat{\phi}, \hat{\psi}, u$, and $v$ are cofibrations, together with a weak equivalence both of CDGA-squares and of $\hat{\phi}$-squares $\hat{\Theta}^{\prime}: \hat{\mathbb{D}} \stackrel{\simeq}{\rightrightarrows} \mathbb{D}^{\prime}$. Moreover $X$ and $Y$ can be chosen such that such that $X^{<n-m-1}=Y^{<n-m-2}=0$. If $H^{1}(f ; \mathbb{Q})$ is injective we can also assume that $Y^{<n-m-1}=0$.

Proof By taking a minimal relative Sullivan algebra of $l^{*} \alpha^{\prime}$ we get a commutative diagram of CDGA

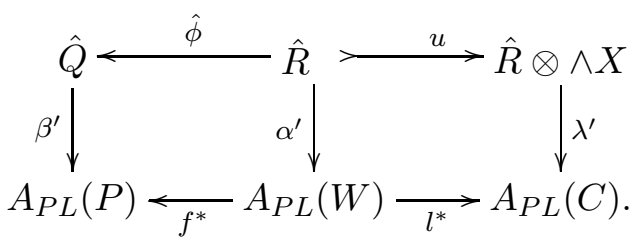

Consider the push-out $\hat{Q} \otimes \wedge X$ of the top line of the above diagram. By the universal property of the push-out, this diagram induces a CDGA map

$$
\bar{\mu}_{0}^{\prime}: \hat{Q} \otimes \wedge X \rightarrow A_{P L}(\partial T) .
$$

The latter map can be factored into a minimal relative Sullivan algebra followed by a quasi-isomorphism,

$$
\hat{Q} \otimes \wedge X \stackrel{v}{\longrightarrow} \hat{Q} \otimes \wedge X \otimes \wedge Y \stackrel{\mu^{\prime}}{\simeq} A_{P L}(\partial T) .
$$

It is immediate to check that the matrix $\hat{\Theta}^{\prime}=\left(\begin{array}{cc}\alpha^{\prime} & \beta^{\prime} \\ \lambda^{\prime} & \mu^{\prime}\end{array}\right)$ is a weak equivalence of CDGA-squares and of $\hat{\phi}$-squares.

We prove now that $X^{<n-m-1}=0$. Since $i$ is $(n-m-1)$-connected a MayerVietoris argument implies that $H_{*}(l)$ is $(n-m-1)$-connected. Therefore the same is true for the map $u$ and by minimality we get that $X^{<n-m-1}=0$.

The model $\hat{Q} \rightarrow \hat{Q} \otimes \wedge X \otimes \wedge Y$ of $i^{*}$ is cohomologically $(n-m-1)$-connected and since $X^{<n-m-1}=0$, minimality implies that $Y^{<n-m-2}=0$. 
Assume that $H^{1}(f)$ is injective. Thus $H^{*}(f)$ is 1 -connected and $H^{*}(l)$ is $(n-m-1)$-connected. By a rational Blackers-Massey argument we deduce that $\hat{Q} \otimes \wedge X \rightarrow \hat{Q} \otimes \wedge X \otimes \wedge Y$ is cohomologically $(n-m-1)$-connected. By minimality we get that $Y^{<n-m-1}=0$.

Lemma 7.11 With the hypotheses of Theorem 1.2 and with the notation of Lemma 7.10, the commutative squares of CDGA $\hat{\mathbb{D}}$ and $\mathbb{D}$ are weakly equivalent.

Proof The proof is in two steps:

(i) We will show that the morphism $\hat{\Theta}^{\prime}$ constructed in Lemma [7.10 lifts along the acyclic fibration $\Theta^{\prime}$ of Lemma 7.9 to a weak equivalence of $\hat{\phi}$-squares $\bar{\Theta}: \hat{\mathbb{D}} \stackrel{\simeq}{\rightrightarrows} \overline{\mathbb{D}}$.

(ii) using the map $\Theta$ constructed in Lemma[7.9 we will show that the composite $\hat{\Theta}:=\Theta \bar{\Theta}$ is a quasi-isomorphism of CDGA.

(i) The lift will be of the form $\bar{\Theta}:=\left(\begin{array}{cc}\rho & \text { id } \\ \bar{\lambda} & \bar{\mu}\end{array}\right)$, where $\rho$ was defined in Lemma 7.2. We need only to build the maps $\bar{\lambda}$ and $\bar{\mu}$, and for this we will use the maps $\lambda^{\prime}$ and $\mu^{\prime}$ constructed in the proof of Lemma 7.10. We have the following solid commutative diagram of $\hat{R}$-DGmodules

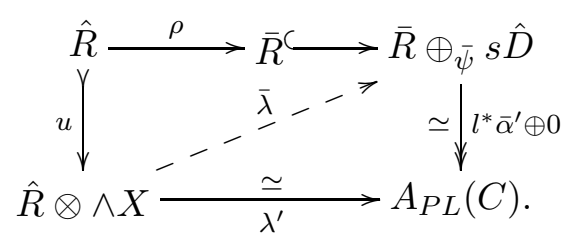

Since $u$ is a relative Sullivan algebra, it is an $\hat{R}$-DGmodule cofibration. Then, $l^{*} \bar{\alpha} \oplus 0$ being an acyclic fibration, there exists a lift of $\hat{R}$-DGmodules, $\bar{\lambda}$, making both triangles of the diagram commute.

We can define a map of $\hat{Q}$-DGmodules

$$
\bar{\mu}_{0}: \hat{Q} \otimes \wedge X \rightarrow \hat{Q} \oplus_{\chi} s \hat{D}
$$

by the formula

$$
\bar{\mu}_{0}(q \otimes \omega)=q \cdot(\bar{\phi} \oplus \mathrm{id})(\bar{\lambda}(1 \otimes \omega)),
$$


for $q \in \hat{Q}$ and $\omega \in \wedge X$. It is immediate to check that $\bar{\mu}_{0}$ is a $\hat{Q}$-DGmodule morphism and that the following solid diagram of $\hat{Q}$-DGmodules commutes:

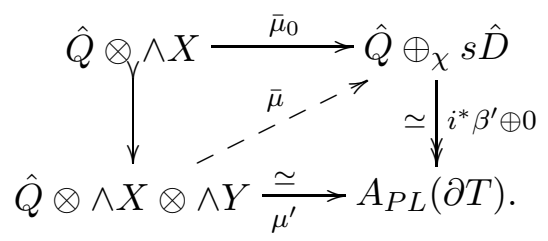

Therefore there exists a lift, $\bar{\mu}$, of $\hat{Q}$-DGmodules making both triangles of the diagram commute.

It is immediate to check that $\bar{\Theta}:=\left(\begin{array}{cc}\rho & \text { id } \\ \bar{\lambda} & \bar{\mu}\end{array}\right)$ is a weak equivalence of $\hat{\phi}$ squares.

(ii) We show now that the composite $\hat{\Theta}:=\Theta \bar{\Theta}: \hat{\mathbb{D}} \rightarrow \mathbb{D}$ is a weak equivalence in the category of commutative squares of CDGA. We know already that $\hat{\Theta}$ is a quasi-isomorphism, since both $\Theta$ and $\bar{\Theta}$ are. Recalling the form of $\Theta$ from the proof of Lemma 7.9 and of $\bar{\Theta}$ from the proof of (i), we see that

$$
\hat{\Theta}=\left(\begin{array}{cc}
\bar{\alpha} & \beta \\
\bar{\alpha} \oplus s \gamma & \beta \oplus s \gamma
\end{array}\right)\left(\begin{array}{cc}
\rho & \mathrm{id} \\
\bar{\lambda} & \bar{\mu}
\end{array}\right)=\left(\begin{array}{cc}
\alpha & \beta \\
\lambda & \mu
\end{array}\right),
$$

where $\alpha, \beta$ are CDGA morphisms and $\lambda$ (resp. $\mu$ ) is some $\hat{R}$-DGmodule (resp. $\hat{Q}$-DGmodule) morphism.

By the hypotheses of Theorem 1.2 we have that $\left(R \oplus_{\psi} s D\right)^{>n}=0$. Since $n \geq 2 m+3$, this implies that $\left(R \oplus_{\psi} s D\right)^{\geq 2(n-m-1)}=0$. Since $X^{<n-m-1}=0$, Lemma 4.5 implies that $\lambda$ is a CDGA morphism.

Suppose that $H^{1}(f)$ is injective and $n \geq 2 m+3$. A similar argument shows that $\mu$ is a CDGA morphism, which implies that $\hat{\Theta}$ is a weak equivalence of squares of CDGA.

Suppose instead that $n \geq 2 m+4$. Since $Q$ is connected and $H^{\geq n}(Q \oplus s D)=0$ there exists an acyclic ideal $L \subset Q \oplus s D$ such that $((Q \oplus s D) / L)^{\geq n}=0$. Replace $Q \oplus s D$ in diagram $\mathbb{D}$ by $(Q \oplus s D) / L$ to get a quasi-)isomorphic CDGA diagram $\tilde{\mathbb{D}}$. Since $Y^{<n-m-2}=0$ and $2(n-m-2) \geq n$, Lemma 4.5] implies that the composite $\hat{Q} \otimes \wedge X \otimes \wedge Y \stackrel{\mu}{\rightarrow} Q \oplus s D \stackrel{\simeq}{\rightarrow}(Q \oplus s D) / L$ is a CDGA quasi-isomorphism. Therefore $\hat{\mathbb{D}} \simeq \tilde{\mathbb{D}} \simeq \mathbb{D}$ as CDGA squares.

Collecting these lemmas we conclude the proof of the first part of the theorem: 
Proof of Theorem 1.2 Lemmas 7.10 and 7.11 imply that the diagrams $\mathbb{D}$ and $\mathbb{D}^{\prime}$ are weakly equivalent CDGA commutative squares.

We prove now the second part of the theorem. Suppose given a CDGA model $\phi_{0}: R_{0} \rightarrow Q_{0}$ of $f^{*}$. Our goal is to build a model $\phi: R \rightarrow Q$ and a top-degree map $\psi: D \rightarrow R$ fulfilling hypotheses (i-)-(iii) of Theorem 1.2. By replacing $\phi_{0}$ by a minimal Sullivan model we can suppose that both $R_{0}$ and $Q_{0}$ are connected. Since $H^{>n-1}\left(Q_{0}\right)=0$ and $H^{>n}\left(R_{0}\right)$ we can build another CDGA model of $f^{*}$ of the form $\phi_{1}: R \rightarrow Q_{1}$ with $R^{>n}=0$, and such that $R$ and $Q_{1}$ are still connected. We can factor $\phi_{1}$ into a minimal relative Sullivan algebra $\phi_{2}$ followed by a weak equivalence. This gives another CDGA model of $f^{*}$ of the form $\phi_{2}: R \longrightarrow Q_{2}:=R \otimes \wedge V$ and $V=V^{\geq 1}$ because $H^{1}(f ; \mathbb{Q})$ is injective.

Let $D_{2}$ be a minimal semifree model of the $Q_{2}$-DGmodule $s^{-n} \# Q_{2}$. Since $H^{<n-m}\left(s^{-n} \# Q_{2}\right)=0$, minimality implies that $D_{2}^{<n-m}=0$. Since $\phi_{2}$ is a relative Sullivan algebra, every semifree $Q_{2}$-DGmodule is also a semifree $R$ DGmodule. Therefore $D_{2}$ is also a cofibrant $R$-DGmodule and Proposition 5.6 implies that there exists a top-degree map of $R$-DGmodule $\psi_{2}: D_{2} \rightarrow R$. Since $H^{>n}\left(s^{-n} \# Q_{2}\right)=0$ we can replace $D_{2}$ by a weakly equivalent $Q_{2}$-DGmodule, $D$, such that $D^{<n-m}=0, D^{>n+1}=0$, and $D^{\leq n}=D_{2}^{\leq n}$. Since $R^{>n}=0$ the map $\psi_{2}$ induces a top-degree map $\psi: D \rightarrow R$. Since $H^{>m}\left(Q_{2}\right)=0$ and $Q_{2}$ is connected there exists a surjective quasi-isomorphism of CDGA $\alpha_{2}: Q_{2} \stackrel{\widetilde{ }}{\rightarrow} Q$ such that $Q^{>m+2}=0$ and $\operatorname{ker}\left(\alpha_{2}\right) \subset Q^{>m+1}$. For degree reasons $\left(\operatorname{ker} \alpha_{2}\right) . D=$ 0 , therefore the $Q_{2}$-DGmodule $D$ inherits a $Q$-DGmodule structure. Set $\phi=$ $\alpha_{2} \phi_{2}$.

In summary we have built from $\phi_{0}$ another CDGA model $\phi$ of $f^{*}$ and a topdegree map of $R$-DGmodule $\psi$ satisfying hypotheses (i)-(iii).

\section{CDGA models of the complement in a Poincaré embedding under the unknotting condition}

In this section we give a proof of Theorem 1.4 which gives a CDGA model of the complement in a Poincaré embedding under the unknotting condition. We also build a model of a diagram which is almost the Poincaré embedding (1.1) under a slightly stronger unknotting condition (Theorem 8.2).

The proof of Theorem 1.4 follows the line of the proof of Theorem 1.2. In particular we will reuse many of the lemmas of the previous section. First it 
is easy to check that if we replace the hypotheses of Theorem 1.2 by those of Theorem 1.4 in Lemmas [7.1 and [7.2 then the conclusions of these lemmas still hold without any change in their proofs. Since $D$ is supposed to be only an $R$-DGmodule model of $s^{-n} \# Q$ we replace Lemma 7.3 by the following

Lemma 8.1 With the hypotheses of Theorem 1.4 and with the notation of Lemmas 7.1 and 7.2, there exists a cofibrant $\hat{R}$-DGmodule, $\hat{D}$, and weak equivalences of $\hat{R}$-DGmodules,

$$
D \stackrel{\gamma}{\simeq} \hat{D} \stackrel{\gamma^{\prime \prime}}{\simeq} A_{P L}(W, C),
$$

making the following diagram of isomorphisms commute

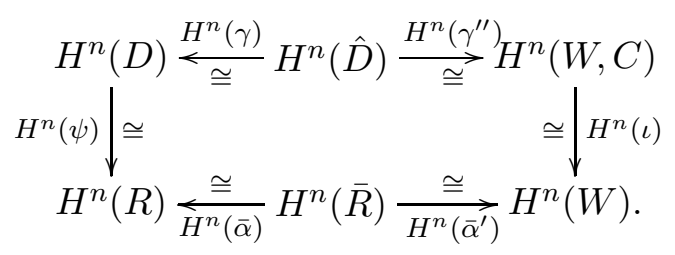

Proof It is a special case of Lemma 6.4

It can be readily checked that Lemma 7.4 still holds when we replace the hypotheses of Theorem 1.2 by those of Theorem 1.4 and the only change in the proof of this lemma is a replacement of the reference to Lemma 7.3 to a reference to Lemma 8.1 Moreover by Lemma 3.6 we can replace $\tilde{\psi}$ by $\bar{\psi}$ making the diagram of Lemma 7.4 strictly commute. We are now ready for the following

Proof of Theorem 1.4 By the same argument as for Theorem 1.2 and using Lemma 7.1, 7.2, 8.1, and 7.4, we get that $R \hookrightarrow R \oplus_{\psi} s D$ is an $\hat{R}$-DGmodule model of $l^{*}: A_{P L}(W) \hookrightarrow A_{P L}(C)$.

Since $H_{*}(f)$ is $r$-connected and by Lefschetz duality we have $H^{\geq n-r}\left(R \oplus_{\psi}\right.$ $s D)=H^{\geq n-r}(C ; \mathbb{Q})=H_{\leq r}(W, P ; \mathbb{Q})=0$. Using the connectivity of $R$ it is easy to build an acyclic subDGmodule $L \subset R \oplus_{\psi} s D$ concentrated in degrees $\geq n-r-1$ and killing $\left(R \oplus_{\psi} s D\right)^{\geq n-r}$.

Consider such an acyclic subDGmodule $L$. Set $k=n-m-1$ and $l=n-2 m+$ $r-1$. By Lemma 4.3 there is a semi-trivial CDGA structure on $\left(R \oplus_{\psi} s D\right) / L$ and the obvious map $R \rightarrow\left(R \oplus_{\psi} s D\right) / L$ is a CDGA morphism. Since $L$ is acyclic the map $R \rightarrow\left(R \oplus_{\psi} s D\right) / L$ is also an $\hat{R}$-DGmodule model $l^{*}$. 
Let $\hat{R} \stackrel{u}{\longrightarrow} \hat{R} \otimes \wedge X \stackrel{\lambda^{\prime}}{\simeq} A_{P L}(C)$ be a CDGA factorization of $l^{*} \alpha^{\prime}$ through a relative minimal Sullivan algebra $\hat{R} \otimes \wedge X$. By the same argument as in the proof of Lemma 7.10 we find that $X^{<n-m-1}=0$ because $l^{*}$ is $(n-m-1)$ connected.

Since $u$ is a model of $\hat{R}$-DGmodule of $l^{*}$, the same argument as in the beginning of the proof of Lemma 7.11 gives a commutative diagram of $\hat{R}$-DGmodules

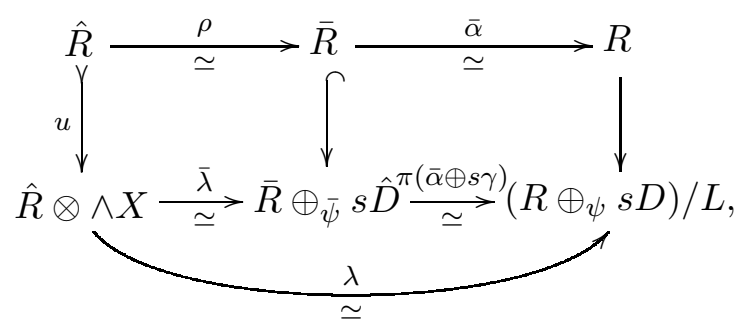

and the composite $\lambda=\pi(\bar{\alpha} \oplus s \gamma) \bar{\lambda}$ is a quasi-isomorphism.

Since $X^{<n-m-1}=0$ and $\left(\left(R \oplus_{\psi} s D\right) / L\right)^{\geq n-r}=0$, the condition $r \geq 2 m-n+2$ and Lemma 4.5 imply that $\lambda$ is a CDGA morphism. Also $\bar{\alpha} \rho=\alpha$ is a CDGA morphism. Thus $u$ is a CDGA model of $R \rightarrow\left(R \oplus_{\psi} s D\right) / L$. By construction $u$ is also a model of $l^{*}$ and the first part of the theorem is proved.

The second part of the theorem is proved in a similar way to Theorem 1.2

Proof of Corollary 1.5 Since $P$ is simply connected and the codimension is at least $3, \partial T$ is simply connected, and since $W$ is also simply-connected, the same is true for $C$ by Van Kampen theorem. The corollary follows then from the above theorem and from the fact that a CDGA model of a simply connected spaces of finite type determines its rational homotopy type.

In the rest of the section we address the problem of describing a CDGA model of Diagram (1.1) under some unknotting condition. We wish that we could have determined the rational homotopy type of the entire square (1.1) from the rational homotopy class of $f$, but we are only able to determine a slightly less complete square that we describe now.

Assume that $\partial T$ is simply-connected in which case by Poincaré duality in dimension $n-1$ and by [28, Proposition 4.1] we can consider the space $\partial \check{T}$ obtained by removing the unique top $(n-1)$-cell in a minimal $\mathrm{CW}$-decomposition 
of $\partial T$. We have then the following commutative square of topological spaces

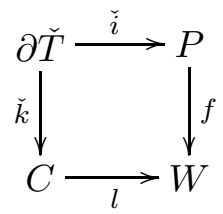

where $\check{i}$ and $\check{k}$ are the restrictions of $i$ and $k$ to $\partial \check{T}$. Our next theorem is a description of a CDGA model of (8.1) under a stronger unknotting condition and two extra assumptions which are not too restrictive as we explain in Remark 8.3.

To state the theorem it is convenient to introduce the following terminology: if $X$ is an $A$-DGmodule and $l$ is an integer then a truncation $A$-subDGmodule of $X$ above degree $l$ is a subDGmodule $L$ such that $L^{\leq l-1}=0, X^{>l} \subset L$ and the projection $\pi: X \rightarrow X / L$ induces an isomorphism in homology in degrees $\leq l$. Of course $(X / L)^{>l}=0$. It is easy to check that such a truncation subDGmodule exists when $A$ is connected.

Theorem 8.2 Consider the diagram (8.1) induced by a Poincaré embedding (1.1) with $P$ and $W$ connected and $\partial T$ simply-connected. Let $r$ be a positive integer such that $\tilde{H}_{\leq r-1}(P ; \mathbb{Q})=\tilde{H}_{\leq r}(W ; \mathbb{Q})=0$ and $r \geq 2 m-n+2$. Let $\phi: R \rightarrow Q$ be a $C D G A$ model of $f^{*}: A_{P L}(W) \rightarrow A_{P L}(P)$ such that $R$ is connected. Let $D$ be an $R$-DGmodule weakly equivalent to $s^{-n} \# Q$ and such that $D^{<n-m}=0$. Suppose given a top-degree map of $R$-DGmodules $\psi: D \rightarrow$ $R$.

Suppose moreover that $n \geq m+r+2$ and that $Q$ is $(r-1)$-connected, that is $Q^{\leq r-1}=Q^{0}=\mathbb{Q}$.

Let $I$ be a truncation $R$-subDGmodule of $R$ above degree $n-r-1$, let $J$ be a truncation $Q$-subDGmodule of $Q$ above degree $m$, and let $K$ be a truncation $Q$-subDGmodule of $D$ above degree $n-r$.

Then the following two commutative squares are weakly equivalent in CDGA

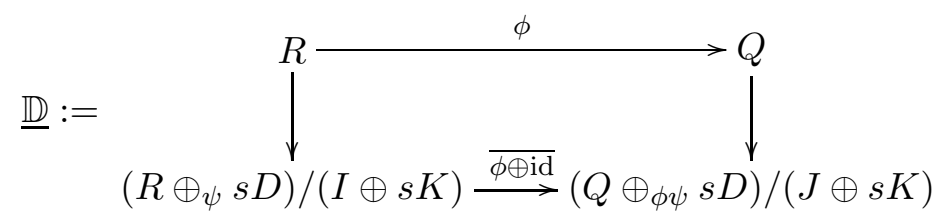


and

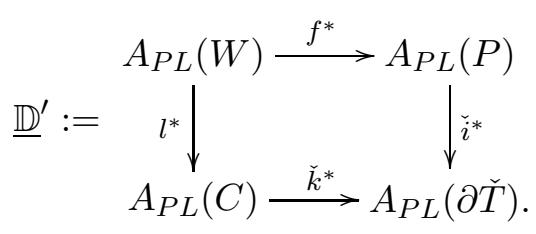

where, in Diagram $\underline{\mathbb{D}}$, the vertical maps are the composition of the inclusion with the projection, the bottom map is the one induced by $\phi \oplus \mathrm{id}_{s D}$, and the CDGA structure on the truncated mapping cones are the semi-trivial ones.

Remark 8.3 The connectivity hypothesis on $P$ and $W$ are equivalent to $H_{*}(f ; \mathbb{Q})$ is $r$-connected and $\tilde{H}_{\leq r-1}(P ; \mathbb{Q})=0$ which is clearly a stronger condition than the unknotting condition (1.3) because of the high connectivity hypothesis on $P$. The first extra assumption in the theorem, $n \geq m+r+2$, is satisfied under the unknotting condition $r \geq 2 m-n+2$ as soon as $m \geq 2 r$. On the other hand, if $m<2 r$ then by a rational version of the suspension Freudenthal theorem, $P$ has the rational homotopy type of a wedge of spheres of dimensions between $r$ and $2 r-1$. Hence this first extra assumption is a consequence of the unknotting condition when $P$ is not rationally equivalent to a wedge of spheres. For the second extra assumption (the $(r-1)$-connectivity of $Q$ ), since $\tilde{H} \leq r(P)=0$, one can always construct an $r$-connected CDGA model $Q$ of $P$, by taking for example a minimal Sullivan model of any given model of $P$. Therefore there is no real loss of generality in making this second assumption.

Remark 8.4 It is very likely that under the only unknotting condition (1.3) one can determine a CDGA model of the complete Poincaré embedding (1.1) but we were unable to prove this.

The rest of the section is devoted to the proof of Theorem 8.2 which is a refinement of the proof of Theorem 1.2 .

Lemmas 7.1 7.4 hold with the hypotheses of Theorem 1.2 replaced by those of Theorem 8.2. We need the following three lemmas in replacement of Lemma 7.5

Lemma 8.5 With the hypotheses of Theorem 8.2 and with the notation of Lemmas 7.1 7.4, the composite $\phi \psi$ induces a $Q$-DGmodule map $\overline{\phi \psi}: D / K \rightarrow$ $Q / J$. There exists a $\hat{Q}-D G m o d u l e$ morphism $\chi: \hat{D} \rightarrow \hat{Q}$ making the following 
diagram commute in $\hat{Q}-D G M o d$

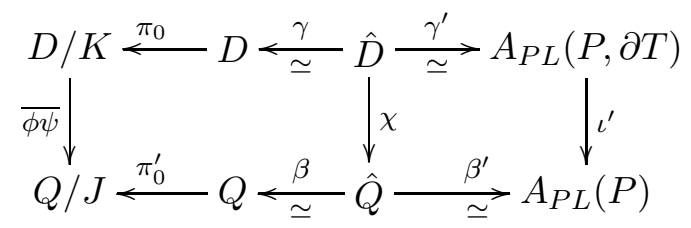

where $\pi_{0}$ and $\pi_{0}^{\prime}$ are the canonical projections. Moreover $\chi \simeq_{\hat{R}} \bar{\phi} \tilde{\psi}$.

Proof Since $n \geq m+r+2$ we have that $\phi \psi(K) \subset J$, hence there is an induced map $\overline{\phi \psi}$ between the quotients. Since $Q$ is $(r-1)$-connected, $(D / K)^{<n-m}=0$, $(Q / J)^{>m}=0$, and $(n-m)+r>m$, we have that the $\mathbf{k}$-DGmodule map $\overline{\phi \psi}$ is a map of $Q$-DGmodule.

Since $r \geq 2 m-n+2$ and $D^{<n-m}=0$, we have $H^{\leq m-r+1}(\hat{D})=0$. Also $\tilde{H}^{\leq r-1}(\hat{Q})=H^{>m}(\hat{Q})=0$. By Lemma [5.7] we have an isomorphism $H^{*}:[\hat{D}, \hat{Q}]_{\hat{Q}} \cong \operatorname{hom}_{\mathbf{k}}^{0}\left(H^{*}(\hat{D}), H^{*}(\hat{Q})\right)$. Therefore there exists a map $\chi: \hat{D} \rightarrow \hat{Q}$ of $\hat{Q}$-DGmodules, unique up to homotopy, such that $H^{*}(\chi)=H^{*}(\bar{\phi} \tilde{\psi})$ where $\bar{\phi}$ and $\tilde{\psi}$ were defined in Lemma 7.2 and Lemma 7.4

Since $\chi$ induces in cohomology the same map as $\bar{\phi} \tilde{\psi}$, Lemma 7.4, Lemma 7.1] and Lemma 7.2 imply that the map $\chi$ makes the diagram of the statement of Lemma 8.5 commute in cohomology. Another application of Lemma [5.7 implies that this diagram commutes up to a homotopy of $\hat{Q}$-DGmodules. Since $\left(\beta \pi_{0}^{\prime}, \beta^{\prime}\right)$ is surjective we can suppose by Lemma 3.6 that $\chi$ makes the diagram exactly commute.

Finally we have also $\chi \simeq_{\hat{R}} \bar{\phi} \tilde{\psi}$, again by Lemma 5.7 .

Lemma 8.6 With the hypotheses of Theorem 8.2 the canonical projection

$$
\pi: R \oplus_{\psi} s D \rightarrow\left(R \oplus_{\psi} s D\right) /(I \oplus s K)
$$

is a quasi-isomorphism and the canonical projection

$$
\pi^{\prime}: Q \oplus_{\phi \psi} s D \rightarrow\left(Q \oplus_{\phi \psi} s D\right) /(J \oplus s K)
$$

induces an isomorphism in cohomology in all degrees except in degree $n-1$ where $H^{n-1}\left(Q \oplus_{\phi \psi} s D\right) \cong \mathbb{Q}$ and $H^{n-1}\left(\left(Q \oplus_{\phi \psi} s D\right) /(J \oplus s K)\right)=0$.

Proof $L=I \oplus s K$ is a truncation $R$-subDGmodule of $R \oplus_{\psi} s D$ above degree $n-r$. Using the fact that $H^{n}(\psi)$ is an isomorphism and that $H^{i}(R)=$ $H^{i}(s D)=0$ for $n-r \leq i \neq n$, it comes that $L$ is acyclic, hence $\pi$ is a quasi-isomorphism. 
The proof for $\pi^{\prime}$ is similar after computing that $H^{\geq n-r}\left(Q \oplus_{\phi \psi} s D\right) \cong s^{-(n-1)} \mathbb{Q}$ and using the assumption $n \geq m+r+2$ to check that $J \oplus s K$ is a differential submodule of $Q \oplus_{\psi} s D$.

Lemma 8.7 With the hypotheses of Theorem 8.2 and with the notation of Lemmas 7.1 7.4 and Lemma 8.5, there exists a cofibration of $\hat{Q}-D G m o d u l e s$

$$
w: \hat{Q} \oplus_{\chi} s \hat{D} \longrightarrow\left(\hat{Q} \oplus_{\chi} s \hat{D}\right) \oplus \hat{Q} \otimes V
$$

and acyclic fibrations of $\hat{Q}$-DGmodules $\epsilon$ and $\epsilon^{\prime}$ making the following diagram commute

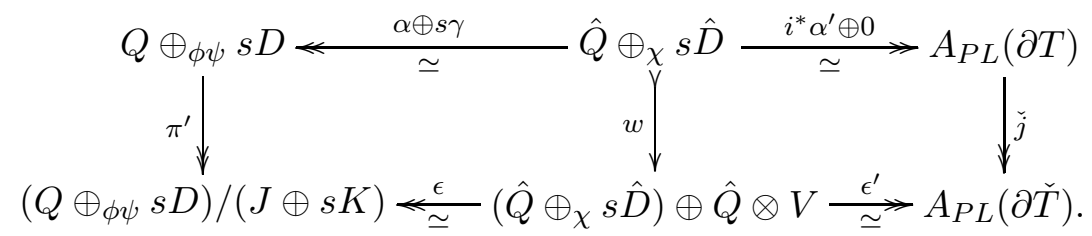

Proof The composite of $\hat{Q}$-DGmodules

$$
\hat{Q} \oplus_{\chi} s \hat{D} \stackrel{i^{*} \alpha^{\prime} \oplus 0}{\simeq} A_{P L}(\partial T) \stackrel{\check{j}}{\longrightarrow} A_{P L}(\partial \check{T})
$$

can de factored into a minimal semi-free extension $w$ followed by a quasiisomorphism $\epsilon^{\prime}$. Moreover, since $\breve{j}\left(i^{*} \alpha^{\prime} \oplus 0\right)$ is a surjection, so is $\epsilon^{\prime}$.

Define $\epsilon$ as the extension of $\pi^{\prime}(\alpha \oplus s \gamma)$ such that $\epsilon(\hat{Q} \otimes V)=0$, which is a $\hat{Q}$-module morphism. It is clear that $\check{j}$ is $(n-2)$-connected and by minimality $V^{<n-2}=0$. Since $r \geq 1$, we have $((Q \oplus s D) /(J \oplus s K))^{\geq n-1}=0$. For degree reasons $\epsilon$ is a DGmodule map.

It remains to prove that $\epsilon$ is a quasi-isomorphism. This is an easy consequence of the fact that $H^{<n-1}\left(\pi^{\prime}\right)$ is an isomorphism and $H^{\geq n-1}\left(\left(Q \oplus_{\phi \psi} s D\right) /(J \oplus s K)\right)=$ $0=H^{\geq n-1}(\partial \check{T})$.

Lemma 7.6 holds with the hypotheses of Theorem 8.2 replacing those of Theorem 1.2 without any change in the proof.

To finish the proof of Theorem [8.2, we adapt the four Lemmas 7.8 7.11] to the setting of this section. Recall Diagrams $\underline{\mathbb{D}}$ and $\underline{\mathbb{D}}^{\prime}$ from the statement of Theorem 8.2 
Lemma 8.8 With the hypotheses of Theorem 8.2 and with the notation of Lemmas 7.1 7.6] and 8.5 8.7. Diagrams $\underline{\mathbb{D}}$ and $\underline{\mathbb{D}}^{\prime}$ are both commutative squares in CDGA and $\hat{\phi}$-squares, and the following diagram is a $\hat{\phi}$-square:

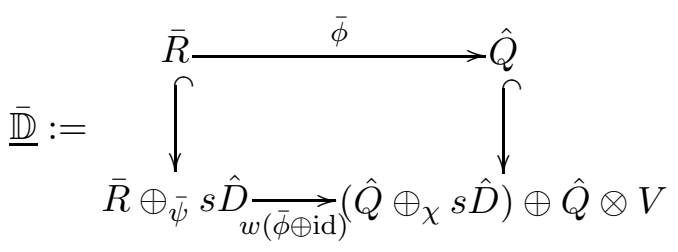

Proof We show first that the $\underline{\mathbb{D}}$ is a diagram of CDGA. We have already shown in the proof of Theorem 1.4 that $R \rightarrow\left(R \oplus_{\psi} s D\right) /(I \oplus s K)$ is a CDGA map. The morphism $\phi \psi$ is not a $Q$-DGmodule morphism but, for degree reasons, the composite $\pi_{0}^{\prime} \phi \psi: D \rightarrow Q / J$ is. Therefore the truncated mapping cone $\left.\left(Q \oplus_{\phi \psi} s D\right) /(J \oplus s K)\right)$ has a natural structure of $Q$-DGmodule. Again by Lemma 4.3] this endows this mapping cone with a semi trivial CDGA structure and the map $Q \rightarrow\left(Q \oplus_{\phi \psi} s D\right) /(J \oplus s K)$ is a CDGA map. Moreover, using the fact that $n \geq m+r+2$ we get that $\phi(I) \subset J$, therefore $\phi \oplus$ id : $R \oplus_{\psi} s D \rightarrow$ $Q \oplus_{\phi \psi} s D$ induces a map, $\overline{\phi \oplus \mathrm{id}}$, between the quotients. It is straightforward to check that it is a CDGA map.

This proves that $\underline{\mathbb{D}}$ is a CDGA square and also a $\hat{\phi}$-square where the $\hat{R}$ - and $\hat{Q}$ DGmodule structures are induced by the maps $\alpha$ and $\beta$. It is immediate that $\underline{\mathbb{D}}^{\prime}$ is a CDGA-square and it is also a $\hat{\phi}$-square where the $\hat{R}$ - and $\hat{Q}$-DGmodule structures are induced by the maps $\alpha^{\prime}$ and $\beta^{\prime}$.

It is immediate that $\underline{\mathbb{D}}$ is a $\hat{\phi}$-square.

Lemma 8.9 With the hypotheses of Theorem 8.2 and with the notation of Lemmas 7.1 7.6 and 8.5 8.8, there exist acyclic fibrations of $\hat{\phi}$-squares

$$
\underline{\mathbb{D}} \underset{\simeq}{\stackrel{\Theta}{\simeq}} \stackrel{\overline{\mathbb{D}}}{\stackrel{\Theta^{\prime}}{\simeq}} \underline{\mathbb{D}}^{\prime}
$$

Proof Set $\Theta=\left(\begin{array}{cc}\bar{\alpha} & \beta \\ \pi(\alpha \oplus s \gamma) & \epsilon\end{array}\right)$ and $\Theta^{\prime}=\left(\begin{array}{cc}\bar{\alpha}^{\prime} & \beta^{\prime} \\ l^{*} \bar{\alpha}^{\prime} \oplus 0 & \epsilon^{\prime}\end{array}\right)$ where $\epsilon$ and $\epsilon^{\prime}$ were defined in Lemma 8.7. An argument analogous to that of Lemma 7.9 together with the results of Lemmas 8.6 and 8.5 finishes the proof. 
Lemma 8.10 With the hypotheses of Theorem 8.2 and with the notation of Lemmas 7.1 7.6 and 8.5 8.9, there exists a commutative square in CDGA,

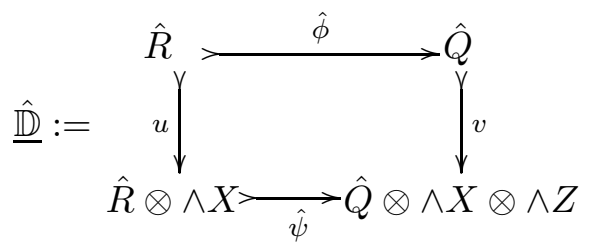

where $\hat{\phi}, \hat{\psi}, u$, and $v$ are cofibrations, and there exists a weak equivalence both of CDGA-squares and of $\hat{\phi}$-squares $\hat{\Theta}^{\prime}: \underline{\mathbb{D}} \stackrel{\simeq}{\rightrightarrows} \underline{\mathbb{D}}^{\prime}$. Moreover $X$ and $Z$ can be chosen such that such that $X^{<n-m-1}=\bar{Z}^{<n-m-1}=0$.

Proof The proof is completely similar to that of Lemma 7.10 replacing $A_{P L}(\partial T)$ by $A_{P L}(\partial \check{T})$, which changes nothing to the $(n-m-1)$-connectivity of the maps and noticing that since $r$ is positive, $H^{1}(f)$ is injective.

Lemma 8.11 With the hypotheses of Theorem 8.2 and with the notation of Lemmas 7.1 7.6 and 8.5 8.10, there exists a quasi-isomorphism of commutative squares in $C D G A \hat{\Theta}: \underline{\hat{\mathbb{D}}} \stackrel{\sim}{\rightrightarrows} \underline{\mathbb{D}}$.

Proof By an completely analogous argument to that of the first part of the proof of Lemma 7.11, we get a lifting of $\hat{\phi}$-squares $\bar{\Theta}: \underline{\mathbb{D}} \stackrel{\simeq}{\rightarrow} \underline{\mathbb{D}}$. It remains then to prove that the composite $\hat{\Theta}:=\bar{\Theta} \hat{\Theta}^{\prime}$ is a morphism of squares of CDGA. This is proved by Lemma 4.5 using the facts that

$$
\left(\left(R \oplus_{\psi} s D\right) /(I \oplus s K)\right)^{\geq n-r}=\left(\left(Q \oplus_{\phi \psi} s D\right) /(J \oplus s K)\right)^{\geq n-r}=0,
$$

that $X^{<n-m-1}=Z^{<n-m-1}=0$, and that $2(n-m-1) \geq n-r$ by (1.3).

Proof of Theorem 1.4 The fact that $\underline{\mathbb{D}}$ is a well defined CDGA square was proved in the first part of Lemma 8.8. Then by Lemmas 8.10] and 8.11, the diagrams $\underline{\mathbb{D}}$ and $\underline{\mathbb{D}}^{\prime}$ are weakly equivalent in CDGA.

\section{Examples of rationally knotted embeddings}

The aim of this section is to show by some examples that the unknotting condition (1.3) in Theorem 1.4 and in the second part of Corollary 1.7 is unavoidable and sharp. Recall that this condition is $r \geq 2 m-n+2$ where

- $m$ is the dimension of the embedded polyhedron $P$, 
- $n-m$ is the codimension of the embedding,

- $r$ is the connectivity of the embedding.

We will build two families of examples where the unknotting condition (1.3) is missed by a little and such that the thesis of Theorem 1.4 does not hold. The unknotting condition can be reformulated as $r+(n-m) \geq m+2$ which can be roughly expressed as

$$
\text { connectivity }+ \text { codimension } \geq \text { dimension }+2 \text {. }
$$

In the first examples that we will build (Proposition 9.1), the connectivity $r$ is big but the codimension $n-m$ is not high enough, and in the second family of examples (Proposition 9.3) the codimension will be big but the connectivity small. Both of these families of examples are fairly explicit and are described in the proof of these propositions.

Proposition 9.1 Let $p$ be a positive even integer and let $n \geq 3 p+2$. Set $m=n-p-1$ and $r=2 m-n+1$. Then there exist two $m$-dimensional polyhedra, $P_{0}$ and $P_{1}$, having both the rational homotopy type of the wedge of spheres $S^{n-2 p-1} \vee S^{n-p-1}$, and two nullhomotopic $r$-connected embeddings $f_{0}: P_{0} \hookrightarrow S^{n}$ and $f_{1}: P_{1} \hookrightarrow S^{n}$, such that the rational cohomology algebras of the complement of these embedded polyhedra are not isomorphic:

$$
H^{*}\left(S^{n} \backslash f_{0}\left(P_{0}\right) ; \mathbb{Q}\right) \varsubsetneqq H^{*}\left(S^{n} \backslash f_{1}\left(P_{1}\right) ; \mathbb{Q}\right) .
$$

Proof Set $X_{0}=S^{p} \vee S^{2 p}$. There exists an obvious PL-embedding $X_{0} \subset S^{n}$. Define $P_{0}$ as the closure of the complement of some regular neighborhood of $X_{0}$ in $S^{n}$. By Lefschetz duality we have $\tilde{H}_{*}\left(P_{0} ; \mathbb{Z}\right)=\mathbb{Z} \cdot x_{n-2 p-1} \oplus \mathbb{Z} \cdot y_{n-p-1}$ and by [28, Proposition 4.1] $P_{0}$ has the homotopy type of a two-cell $\mathrm{CW}$-complex $P_{0} \simeq S^{n-2 p-1} \cup e^{n-p-1}$. Since $n \geq 3 p+2$, we have that $\pi_{n-p-2}\left(S^{n-2 p-1}\right) \otimes \mathbb{Q}=0$ and $P_{0} \simeq_{\mathbb{Q}} S^{n-2 p-1} \vee S^{n-p-1}$. Therefore the rational cohomology algebra $H^{*}\left(S^{n} \backslash P_{0}, \mathbb{Q}\right) \cong H^{*}\left(X_{0} ; \mathbb{Q}\right)$ has a trivial multiplication.

On the other hand consider a $(p-1)$-connected and $2 p$-dimensional polyhedron $X_{1}$ having the homotopy type of the CW-complex $S^{p} \cup_{2[\iota, \iota]} e^{2 p}$ where $\iota \in$ $\pi_{p}\left(S^{p}\right)$ represents the identity map and $[\iota, \iota]$ is the Whitehead bracket. Then $H^{*}\left(X_{1} ; \mathbb{Q}\right) \cong \mathbb{Q}[x] /\left(x^{3}\right)$ with $\operatorname{deg}(x)=p$. By the embedding theorem of Wall [29], after replacing $X_{1}$ by some polyhedron of the same homotopy type, there exists an embedding $X_{1} \subset S^{n}$. Define $P_{1}$ as the closure of the complement of a regular neighborhood of $X_{1}$ in $S^{n}$. By the same argument as for $P_{0}$ we see that $P_{1}$ has the rational homotopy type of the same wedge of spheres. But here the multiplication on the cohomology algebra $H^{*}\left(S^{n} \backslash P_{1} ; \mathbb{Q}\right) \cong H^{*}\left(X_{1} ; \mathbb{Q}\right)$ is not trivial. 
Finally it is immediate that both embeddings $P_{0} \subset S^{n}$ and $P_{1} \subset S^{n}$ are nullhomotopic and $r$-connected.

The previous proposition implies that there is no way of getting a model of the rational homotopy type of the complement $S^{n} \backslash f_{i}\left(P_{i}\right)$ from just a model of the homotopy class of the embedding $f_{i}$. Notice that the equation $r=2 m-n+1$ is very close to the unknotting condition (1.3), showing that this condition is sharp in Theorem 1.4 and Corollary 1.7

Note also that using Spanier-Whitehead duality or the techniques of [22, it can be shown that the two polyhedra $P_{0}$ and $P_{1}$ constructed in Proposition 9.1 can be chosen as having the same integral homotopy type. It is even possible that $P_{0}$ and $P_{1}$ might be chosen as being PL-homeomorphic, but we have no proof of that fact.

The examples of Proposition 9.1 show that the unknotting condition of Theorem 1.4 is sharp at least when the codimension $n-m$ is low (even if the connectivity $r$ is high). In the rest of this section we will build a second family of examples for which the codimension is high but the connectivity is low. We prove first a lemma.

Lemma 9.2 Let $i: X \hookrightarrow S^{n-1}$ be the inclusion of a polyhedron in a sphere and denote by $\epsilon: S^{n-1} \hookrightarrow S^{n}$ the inclusion of the equator. Then

$$
S^{n} \backslash \epsilon(i(X)) \simeq \Sigma\left(S^{n-1} \backslash i(X)\right) .
$$

Proof Set $Y=S^{n-1} \backslash i(X)$. It is clear that the complement of $X$ in $S^{n}$ has the homotopy type of two disks $D^{n}$ glued along $Y \subset S^{n-1}=\partial D^{n}$. Thus

$$
S^{n} \backslash i(X) \simeq D^{n} \cup_{Y} D^{n} \simeq \Sigma Y .
$$

Proposition 9.3 For $0 \leq r \leq 5$ there exists two $r$-connected homotopic embeddings $f_{k}: S^{r} \times S^{7} \hookrightarrow S^{15}, k=0,1$, such that the rational cohomology algebras of their complement are not isomorphic,

$$
H^{*}\left(S^{15} \backslash f_{0}\left(S^{r} \times S^{7}\right) ; \mathbb{Q}\right) \not H^{*}\left(S^{15} \backslash f_{1}\left(S^{r} \times S^{7}\right) ; \mathbb{Q}\right) .
$$

Proof We have the standard embeddings $S^{r} \subset \mathbb{R}^{r+1}$ and $S^{7} \subset \mathbb{R}^{8}$, as well as the "stereographic" embedding $\mathbb{R}^{r+9} \subset\left(\mathbb{R}^{r+9} \cup\{\infty\}\right) \cong S^{r+9}$. Composing those we get an embedding

$$
i: S^{r} \times S^{7} \hookrightarrow \mathbb{R}^{r+1} \times \mathbb{R}^{8}=\mathbb{R}^{r+9} \hookrightarrow S^{r+9} .
$$


Since $r+9 \leq 14$, we have the inclusion of a subequator

$$
\epsilon: S^{r+9} \subset S^{15} \text {. }
$$

Set $f_{0}=\epsilon i$. Lemma 9.2 implies that $S^{15} \backslash f_{0}\left(S^{r} \times S^{7}\right)$ has the homotopy type of a suspension. Therefore the multiplication on $H^{*}\left(S^{15} \backslash f_{0}\left(S^{r} \times S^{7}\right)\right.$; $\left.\mathbb{Q}\right)$ is trivial.

We construct now another embedding $f_{1}$. Consider the Hopf fibration

$$
S^{7} \rightarrow S^{15} \stackrel{\pi}{\rightarrow} S^{8} .
$$

Consider the inclusion of $S^{r}$ in $S^{8}$ as a subequator. Its complement $S^{8} \backslash S^{r}$ has the homotopy type of $S^{7-r}$. Therefore the sphere $S^{15}$ is the union of two polyhedra of the homotopy type of $\pi^{-1}\left(S^{r}\right)$ and $\pi^{-1}\left(S^{7-r}\right)$. Since both of the inclusions $S^{r} \subset S^{8}$ and $S^{7-r} \subset S^{8}$ are nullhomotopic, the restrictions of the Hopf fibration to these subspaces are trivial, hence $\pi^{-1}\left(S^{r}\right) \simeq S^{r} \times S^{7}$ and $\pi^{-1}\left(S^{7-r}\right) \simeq S^{7-r} \times S^{7}$. This defines an embedding $f_{1}: S^{r} \times S^{7} \hookrightarrow S^{15}$ whose complement has the homotopy type of $S^{7-r} \times S^{7}$. Therefore the multiplication on the cohomology algebra $H^{*}\left(S^{15} \backslash f_{1}\left(S^{r} \times S^{7}\right) ; \mathbb{Q}\right)$ is not trivial.

Finally it is immediate that the embeddings $f_{0}$ and $f_{1}$ are homotopic since there are both nullhomotopic for dimension-connectivity reasons.

Taking $r=0$ in Proposition 9.3 gives an example of two homotopic 0 -connected embeddings of $S^{0} \times S^{7}$ in $S^{15}$, of relatively high codimension, and whose complement do not have the same rational homotopy type. Again this shows that the unknotting condition (1.3) is sharp since here $r=2 m-n+1$. Note that $r=0$ is not a positive integer and $P=S^{0} \times S^{7}$ is not connected as it should be in the hypotheses of Theorem 1.4. But if we take $r=1$ we get two 1-connected homotopic embeddings of $S^{1} \times S^{7}$ into $S^{15}$, and the unknotting condition is only missed by 2 in that case.

Examples analogous to those of Proposition 9.3 can be build in other dimensions by replacing the Hopf fibration $S^{7} \rightarrow S^{15} \rightarrow S^{8}$ by the Stiefel fibration

$$
S^{2 k-1} \rightarrow V_{2}\left(\mathbb{R}^{2 k+1}\right) \stackrel{\pi^{\prime}}{\rightarrow} S^{2 k}
$$

where $V_{2}\left(\mathbb{R}^{2 k+1}\right)$ can be seen as the spherical tangent bundle of $S^{2 k}$. Since the Euler characteristic of an even-dimensional sphere is not zero, it is immediate that $V_{2}\left(\mathbb{R}^{2 k+1}\right)$ has the rational homotopy type of a sphere $S^{4 k-1}$. We leave to the reader the details of the statement and proof of a proposition analogous to 9.3 with two embeddings of $S^{r} \times S^{2 k-1}$ into $V_{2}\left(\mathbb{R}^{2 k+1}\right) \simeq_{\mathbb{Q}} S^{4 k-1}$ for which the rational cohomology algebras of the complements are not isomorphic. 


\section{References}

[1] C. Boilley, Extensions de modules et dualité de Lefschetz, In preparation.

[2] A.K. Bousfield, V.K.A.M. Gugenheim, On PL DeRham theory and rational homotopy type, Mem. Amer. Math. Soc. 8 (1976) 179 MathReview

[3] G.E. Bredon, Topology and Geometry, Corrected third printing, Graduate Texts in Mathematics 139, Springer-Verlag (1997) MathReview

[4] P. Deligne, Théorie de Hodge, II, Publ. Math. I.H.E.S. 40 (1971) 5-58 MathReview

[5] W.G. Dwyer, J. Spalinski, Homotopy theories and model categories, In: Handbook of Algebraic Topology, edited by I.M. James, Elsevier Science B.V. (1995) 73-126 MathReview

[6] Y. Félix, S. Halperin, J.C. Thomas, Gorenstein spaces, Advances in Math 71 (1988) 92-112 MathReview

[7] Y. Félix, S. Halperin, J.C. Thomas, Differential graded algebras in topology, In: Handbook of Algebraic Topology, edited by I.M. James, Elsevier Science B.V. (1995) 829-865 MathReview

[8] Y. Félix, S. Halperin, J.C. Thomas, Rational Homotopy Theory, Graduate Texts in Mathematics 205, Springer-Verlag (2001) MathReview

[9] C. Gordon, J. Luecke, Knots are determined by their complements, J. Amer. Math. Soc. 2 (1989) 371-415 MathReview

[10] M. Hovey, Model categories, Mathematical Surveys and Monographs 63, American Mathematical Society, Providence, RI (1999) MathReview

[11] S. Halperin, Lectures on minimal models, Mémoires de la Société mathématique de France, 230 (1983) MathReview

[12] S. Halperin, J.M. Lemaire, Notion of category in differential algebra, In: Algebraic Topology - Rational Homotopy, Springer Lecture Notes in Mathematics 1318, Berlin-Heidelberg-New-York: Springer-Verlag (1988) 138-154 MathReview

[13] J.F.P. Hudson, Concordance, isotopy, and diffeotopy, Annals of Math. 91 (1970) 425-448 MathReview

[14] T. Kahl, P. Lambrechts, L. Vandembroucq, Modèles de Quillen des bords homotopiques, submitted, available at: http://www.math.ucl.ac.be/membres/lambrechts/articles.html

[15] J. Klein Poincaré duality spaces, In: Surveys on surgery theory, Vol. 1, Ann. of Math. Stud. 145, Princeton Univ. Press, Princeton, NJ (2000) 135-165 MathReview

[16] J. Klein Poincaré duality embeddings and fiberwise homotopy theory, II, Quart. Jour. Math. Oxford 53 (2002) 319-335 MathReview 
[17] J. Klein Embedding, compression and fiberwise homotopy theory, Algebr. Geom. Topol. 2 (2002) 311-336 MathReview

[18] P. Lambrechts, Cochain models of thickenings and its application to rational LS-category, Manuscripta Math. 103 (2000) 143-160 MathReview

[19] P. Lambrechts, D. Stanley, Examples of rational homotopy types of blowups, Proc. American Math. Soc. (to appear), available at: http://www.math.ucl.ac.be/membres/lambrechts/articles.html

[20] P. Lambrechts, D. Stanley, The rational homotopy type of configuration spaces of two points, Annales Inst. Fourier. 54 (2004) 1029-1052 MathReview

[21] P. Lambrechts, D. Stanley, The rational homotopy type of a blow-up in the stable range, submitted, available at: http://www.math.ucl.ac.be/membres/lambrechts/articles.html

[22] P. Lambrechts, D. Stanley, L. Vandembroucq, Embeddings up to homotopy type of two-cones in Euclidean space, Trans. American Math. Soc. 354 (2002) 3973-4013 MathReview

[23] N. Levitt, On the structure of Poincaré duality spaces, Topology 7 (1968) 369388 MathReview

[24] R. Lickorish, An introduction to knot theory, Graduate Texts in Mathematics 175, Springer-Verlag (1997) MathReview

[25] J. Morgan, The algebraic topology of smooth algebraic varieties, Publ. Math. I.H.E.S. 48 (1978) 137-204 MathReview

[26] C.P. Rourke, B.J. Sanderson, Introduction to Piecewise-Linear Topology, Springer Study Edition, Springer-Verlag, Berlin-Heidelberg-New-York (1982) MathReview

[27] D. Sullivan, Infinitesimal computations in topology, Publ. Math. I.H.E.S. 47 (1977) 269-331 MathReview

[28] C.T.C. Wall, Finiteness conditions for $C W$-complexes, Annals of Math. 81 (1965) 56-69 MathReview

[29] C.T.C. Wall, Classification problems in differential topology - IV. Thickenings, Topology 5 (1966) 73-94 MathReview

Institut Mathématique, Université de Louvain, 2, chemin du Cyclotron

B-1348 Louvain-la-Neuve, Belgium

Department of Mathematics and Statistics, University of Regina

College West 307.14, Regina, Saskatchewan, Canada, S4S 0A2

Email: lambrechts@math.ucl.ac.be and stanley@math.uregina.ca

Received: 8 May 2003 Revised: 18 August 2004 